\title{
Symmetric Implication Zroupoids and Weak Associative Laws
}

\author{
Juan M. Cornejo and Hanamantagouda P. Sankappanavar
}

\begin{abstract}
An algebra $\mathbf{A}=\langle A, \rightarrow, 0\rangle$, where $\rightarrow$ is binary and 0 is a constant, is called an implication zroupoid ( $\mathcal{I}$-zroupoid, for short) if $\mathbf{A}$ satisfies the identities: $(x \rightarrow y) \rightarrow z \approx\left(\left(z^{\prime} \rightarrow x\right) \rightarrow(y \rightarrow z)^{\prime}\right)^{\prime}$ and $0^{\prime \prime} \approx 0$, where $x^{\prime}:=x \rightarrow 0$. An implication zroupoid is symmetric if it satisfies: $x^{\prime \prime} \approx x$ and $\left(x \rightarrow y^{\prime}\right)^{\prime} \approx\left(y \rightarrow x^{\prime}\right)^{\prime}$. The variety of symmetric $\mathcal{I}$ zroupoids is denoted by $\mathcal{S}$. We began a systematic analysis of weak associative laws (or identities) of length $\leq 4$ in [CS18a], by examining the identities of Bol-Moufang type, in the context of the variety $\mathcal{S}$.

In this paper we complete the analysis by investigating the rest of the weak associative laws of length $\leq 4$ relative to $\mathcal{S}$. We show that, of the (possible) 155 subvarieties of $\mathcal{S}$ defined by the weak associative laws of length $\leq 4$, there are exactly 6 distinct ones. We also give an explicit description of the poset of the (distinct) subvarieties of $\mathcal{S}$ defined by weak associative laws of length $\leq 4$.
\end{abstract}

\section{Introduction}

Bernstein, in [Be34], gave a system of axioms for Boolean algebras using implication as the only connective. His system, while not equational, could easily be modified into an equational one by using an additional constant. In 2012, the second author of this paper extended this "modified Bernstein's theorem" to De Morgan algebras (see [San12]). Indeed, he showd in [San12] that the varieties of De Morgan algebras, Kleene algebras, and Boolean algebras are term-equivalent, respectively, to the varieties, $\mathcal{D} \mathcal{M}, \mathcal{K} \mathcal{L}$, and $\mathcal{B A}$ (defined below) whose defining axioms use only an implication $\rightarrow$ and a constant 0 . 
The essential role played by the identity $(\mathrm{I}):(x \rightarrow y) \rightarrow z \approx\left(\left(z^{\prime} \rightarrow x\right) \rightarrow\right.$ $\left.(y \rightarrow z)^{\prime}\right)^{\prime}$, where $x^{\prime}:=x \rightarrow 0$, in the axiomatization of $\mathcal{D} \mathcal{M}, \mathcal{K} \mathcal{L}$, and $\mathcal{B} \mathcal{A}$ motivated the second author to introduce a new equational class of algebras called "implication zroupoids" in [San12].

DEFINITION 1.1. An algebra $\mathbf{A}=\langle A, \rightarrow, 0\rangle$, where $\rightarrow$ is binary and 0 is a constant, is called a zroupoid. A zroupoid $\mathbf{A}=\langle A, \rightarrow, 0\rangle$ is an implication zroupoid ( $\mathcal{I}$-zroupoid, for short) if A satisfies:

(I) $(x \rightarrow y) \rightarrow z \approx\left(\left(z^{\prime} \rightarrow x\right) \rightarrow(y \rightarrow z)^{\prime}\right)^{\prime}$, where $x^{\prime}:=x \rightarrow 0$,

$\left(\mathrm{I}_{0}\right) 0^{\prime \prime} \approx 0$.

$\mathcal{I}$ denotes the variety of implication zroupoids. It is also known that this new variety $\mathcal{I}$ contains the variety $\mathcal{S} \mathcal{L}$ (defined below) which is term-equivalent to the variety of $\vee$-semilattices with the least element 0 (see [CS16a], wherein the name "implicator groupoid" is used instead of "implication zroupoid").

The varieties $\mathcal{D} \mathcal{M}$ and $\mathcal{S} \mathcal{L}$ are defined relative to $\mathcal{I}$, respectively, by the following identities:

(DM) $\quad(x \rightarrow y) \rightarrow x \approx x$ (De Morgan Algebras);

(SL) $\quad x^{\prime} \approx x$ and $x \rightarrow y \approx y \rightarrow x$.

The varieties $\mathcal{K} \mathcal{L}$ and $\mathcal{B} \mathcal{A}$ are defined relative to $\mathcal{D} \mathcal{M}$, respectively, by the following identities:

(KL) $\quad(x \rightarrow x) \rightarrow(y \rightarrow y) \approx y \rightarrow y$ (Kleene algebras);

(BA) $x \rightarrow x \approx 0^{\prime}$ (Boolean algebras).

According to [San12], the variety $\mathcal{I}$ generalizes the variety of De Morgan algebras and also exhibits some interesting properties; for example, the identity $x^{\prime \prime \prime} \rightarrow y \approx x^{\prime} \rightarrow y$ holds in $\mathcal{I}$. Several new subvarieties of $\mathcal{I}$ are also introduced and investigated in [San12]. The (largely unexplored) lattice of subvarieties of $\mathcal{I}$ seems to be fairly complex. In fact, Problem 6 of [San12] calls for an investigation of the structure of the lattice of subvarieties of $\mathcal{I}$.

The papers [CS16a], [CS16b], [CS16c], [CS17], [CS18a], [CS18b], [CS18c] and [GSV] have addressed further, but still partially, the above-mentioned problem by introducing new subvarieties of $\mathcal{I}$ and investigating relationships among them. It follows from [GSV] that the lattice of subvarieties of $\mathcal{I}$ is not modular, whcih answers Problem 5 of [San12] in the negative. The size 
of the poset of subvarieties of $\mathcal{I}$ is known to be at least 40 (as of October 24, 2018); but it is still unknown whether the lattice of subvarieties is finite or infinite.

Given an $\mathcal{I}$-zroupoid $\mathbf{A}$, there are two operations $\wedge$ and $\vee$ on $A$ defined as follows: $x \wedge y:=\left(x \rightarrow y^{\prime}\right)^{\prime}$ and $x \vee y:=\left(x^{\prime} \wedge y^{\prime}\right)^{\prime}$. These operations give rise to the (derived) algebra $\langle A, \wedge, \vee, 0\rangle$ which has been investigated in [CS16a] and [CS17]. Among the important subvarieties of $\mathcal{I}$, the two that are the most relevant to this paper are: $\mathcal{I}_{2,0}$ and $\mathcal{M C}$ which are defined relative to $\mathcal{I}$, respectively, by the following identities:

$$
\begin{aligned}
& \left(\mathrm{I}_{2,0}\right) x^{\prime \prime} \approx x \\
& (\mathrm{MC}) x \wedge y \approx y \wedge x, \text { where } x \wedge y:=\left(x \rightarrow y^{\prime}\right)^{\prime} .
\end{aligned}
$$

We are now ready to make the following definition which plays a fundamental role in the rest of this paper.

DEFINITION 1.2. Let $\mathbf{A} \in \mathcal{I}$. $\mathbf{A}$ is involutive if $\mathbf{A} \in \mathcal{I}_{2,0}$. $\mathbf{A}$ is meetcommutative if $\mathbf{A} \in \mathcal{M C}$. $\mathbf{A}$ is symmetric if $\mathbf{A}$ is both involutive and meetcommutative. Let $\mathcal{S}$ denote the variety of symmetric $\mathcal{I}$-zroupoids. In other words, $\mathcal{S}=\mathcal{I}_{2,0} \cap \mathcal{M C}$.

The investigations in [CS16a] and [CS17] reveal that the variety $\mathcal{S}$ has some interesting properties; for example, for $\mathbf{A} \in \mathcal{I}_{2,0} \cap \mathcal{M C}$, the (derived) algebra $\langle A, \wedge, \vee, 0\rangle$, where $x \vee y:=\left(x^{\prime} \wedge y^{\prime}\right)^{\prime}$, is both a distributive bisemilattice and a Birkhoff system (i.e., satisfies $x \wedge(x \vee y) \approx x \vee(x \wedge y))$. (The name "symmetric $\mathcal{I}$-zroupoids" for the members of $\mathcal{I}_{2,0} \cap \mathcal{M C}$ is new.)

In the present paper we continue our investigations into $\mathcal{S}$. More precisely, we are interested in the subvarieties of $\mathcal{S}$ defined by weak associative laws. A precise definition of a weak associative law appears in [Ku96], which is essentially restated below in our terminology.

DEFINITION 1.3. Let $n \in \mathbb{N}$ and let $\mathcal{L}:=\langle x\rangle$, where $\times$ is a binary operation symbol. Let $p$ be a (groupoid) term in the language $\mathcal{L}$. Then $\operatorname{Var}(p)$ denotes the set of distinct variables occurring in $p . \quad p$ is of length $n$ if the number of (not necessarily distinct) occurrences of variables (in $p$ ) is $n$. An identity $p \approx q$ is said to be a weak associative law of length $n$ in $\mathcal{L}$ if the following conditions hold:

(1) $p$ and $q$ are terms of length $n$;

(2) $\operatorname{Var}(p)=\operatorname{Var}(q)$;

(3) the variables in $p$ and $q$ occur in the same order (only the bracketings are possibly different). (In other words, $p$ and $q$ are built from the same word using (possibly) different bracketings.) 
The following (general) problem was raised in [CS18a]. (In the sequel we use the words "law" and "identity" interchangeably).

PROBLEM: Let $\mathcal{V}$ be a given variety of algebras (whose language includes a binary operation symbol, say, $\times$ ). Investigate the subvarieties of $\mathcal{V}$ defined by weak associative laws (with respect to $\times$ ) and their mutual relationships.

Special cases of the above problem have already been considered in the literature, wherein the weak associative laws chosen are the identities of Bol-Moufang type (i.e., weak associative laws of length 4 with 3 distinct variables), and the variety $\mathcal{V}$ is chosen to be the variety of quasigroups or the variety of loops (for more information about these identities in the context of quasigroups and loops see [Fe69], [Ku96], [PV05a], [PV05b]).

Let $W$ denote the set of identities of weak associative laws of size $\leq 4$. The systematic notation given in the next definition for the identities in $W$ is influenced by the notation developed in [PV05a] for Bol-Moufang identities.

Without loss of generality, we will assume that the variables in the terms $t_{1}$ and $t_{2}$ occur alphabetically in any weak associative identity $t_{1} \approx t_{2}$. Given a word $X$ of variables, we refer to each possible way of bracketing $\mathrm{X}$ that will transform $X$ into a term, as a "bracketing"; and we assign a number to each such bracketing and call it the "bracketing number" of that term.

We will now develop a notation for weak associative laws that helps us to investigate them.

DEFINITION 1.4. Let $n, m, p, q \in \mathbb{N}$ and let $X$ denote a word of length $n$ in which there are $m$ distinct variables occurring alphabetically (with some variables possibly repeated). We denote by (nmXpq) the weak associative identity $t_{1} \approx t_{2}$ of length $n$, with $m$ distinct variables, whose terms $t_{1}$ and $t_{2}$ are obtained from $X$ and have $p$ and $q$ as their respective bracketing numbers. We denote by $n m \mathcal{X} p q$ the variety defined, relative to $\mathcal{S}$, by the weak associative identity (nmXpq).

EXAMPLE 1.5. Let $X$ be the word $\mathrm{A}:=\langle x, x, x\rangle$ where $n=3$ and $m=1$. Then there are two possible brackettings, numbered 1 and 2 , with $a$ as a place holder:

1: $a \rightarrow(a \rightarrow a)$, and $2:(a \rightarrow a) \rightarrow a$. Thus, (31A12) denotes the identity $x \rightarrow(x \rightarrow x) \approx(x \rightarrow x) \rightarrow x$ of length 3 with one variable, and with bracketing numbers 1 and 2 , and $31 \mathcal{A} 12$ denotes the subvariety of $\mathcal{I}$ defined by (31A12), relative to $\mathcal{S}$. 
Here is a second example: Let $X$ be the word $\mathrm{B}:=\langle x, y, x, z\rangle$ where $n=4$ and $m=3$. Then (43B23) denotes the identity $x \rightarrow((y \rightarrow x) \rightarrow z) \approx$ $(x \rightarrow y) \rightarrow(x \rightarrow z)$ of length 4 with 3 distinct variables and with bracketting numbers 2 and 3 in the listing of possible brackettings given in Section 6 below.

Observe that Bol-Moufang identities are precisely the weak associative laws of the form (43Xpq) of length 4 that have 3 distinct variables. It was shown in [CS18a] that there are 4 nontrivial subvarieties of the variety $\mathcal{S}$ of Bol-Moufang type that are distinct from each other (out of the possible 60 varieties), giving the Hasse diagram of the poset formed by them, together with the variety $\mathcal{B A}$ (which is contained in some of them).

In this paper we will complete that analysis by examining the rest of the weak associative laws of length $\leq 4$, relative to $\mathcal{S}$. Clearly, $x \approx x$ and $x \rightarrow y \approx x \rightarrow y$ are the only identitities of length 1 and 2 respectively, which are trivial. So, we will consider the identities of length 3 and 4. In Section 8 , we show, as our main result, that, of the (possible) 155 subvarieties of $\mathcal{S}$, each defined by a single weak associative law of length $\leq 4$, there are exactly 6 distinct ones. We also give an explicit description, by means of a Hasse diagram, of the poset of the (distinct) subvarieties of $\mathcal{S}$ defined by weak associative laws of length $\leq 4$.

We would like to acknowledge that the software "Prover 9/Mace 4" developed by McCune [Mc] has been useful to us in some of our findings presented in this paper. We have used it to find examples and to check some conjectures.

\section{Preliminaries and Properties of $\mathcal{S}$}

We refer the reader to the standard references [BD74], [BS81] and [R74] for concepts and results used, but not explained, in this paper.

Recall from [San12] that $\mathcal{S} \mathcal{L}$ is the variety of semilattices with a least element 0. It was shown in [CS16a] that $\mathcal{S} \mathcal{L}=\mathcal{C} \cap \mathcal{I}_{1,0}$ where the subvarieties $\mathcal{C}$ and $\mathcal{I}_{1,0}$ of $\mathcal{I}$ are defined, respectively, by the identities $x \rightarrow y \approx y \rightarrow x$ and $x^{\prime} \approx x$.

The two-element algebras $\mathbf{2}_{\mathbf{s}}, \mathbf{2}_{\mathrm{b}}$ were introduced in [San12]. Their operations $\rightarrow$ are respectively as follows: 


\begin{tabular}{r|rr}
$\rightarrow:$ & 0 & 1 \\
\hline 0 & 0 & 1 \\
1 & 1 & 1
\end{tabular}

\begin{tabular}{r|rr}
$\rightarrow:$ & 0 & 1 \\
\hline 0 & 1 & 1 \\
1 & 0 & 1
\end{tabular}

Recall that $\mathcal{V}\left(\mathbf{2}_{\mathbf{b}}\right)=\mathcal{B A}$, and $\mathcal{V}\left(\mathbf{2}_{\mathbf{s}}\right)=\mathcal{S L}([$ CS16a, Corollary 10.4] $)$.

LEMMA 2.1. [CS16a] $\mathcal{M C} \cap \mathcal{I}_{1,0} \subseteq \mathcal{C} \cap \mathcal{I}_{1,0}=\mathcal{S L}$.

LEMMA 2.2. [San12, Theorem 8.15] Let $\mathbf{A}$ be an $\mathcal{I}$-zroupoid. Then the following are equivalent:

1. $0^{\prime} \rightarrow x \approx x$,

2. $x^{\prime \prime} \approx x$,

3. $\left(x \rightarrow x^{\prime}\right)^{\prime} \approx x$,

4. $x^{\prime} \rightarrow x \approx x$.

Recall that $\mathcal{I}_{2,0}$ and $\mathcal{M C}$ are the subvarieties defined, respectivaly, relative to $\mathcal{I}$ by the equations

$$
\begin{gathered}
x^{\prime \prime} \approx x . \\
x \wedge y \approx y \wedge x .
\end{gathered}
$$

LEMMA 2.3. [San12] Let $\mathbf{A} \in \mathcal{I}_{2,0}$. Then
1. $x^{\prime} \rightarrow 0^{\prime} \approx 0 \rightarrow x$
2. $0 \rightarrow x^{\prime} \approx x \rightarrow 0^{\prime}$.

LEMMA 2.4. Let $\mathbf{A} \in \mathcal{I}_{2,0}$. Then $\mathbf{A}$ satisfies:

1. $\left(x \rightarrow 0^{\prime}\right) \rightarrow y \approx\left(x \rightarrow y^{\prime}\right) \rightarrow y$,

2. $((0 \rightarrow x) \rightarrow y) \rightarrow x \approx y \rightarrow x$,

3. $\left(x \rightarrow(y \rightarrow x)^{\prime}\right)^{\prime} \approx(x \rightarrow y) \rightarrow x$,

4. $(y \rightarrow x) \rightarrow y \approx(0 \rightarrow x) \rightarrow y$,

5. $(0 \rightarrow x) \rightarrow(x \rightarrow y) \approx x \rightarrow(x \rightarrow y)$,

6. $(0 \rightarrow x) \rightarrow(0 \rightarrow y) \approx x \rightarrow(0 \rightarrow y)$, 


$$
\begin{aligned}
& \text { 7. } x \rightarrow y \approx x \rightarrow(x \rightarrow y), \\
& \text { 8. } 0 \rightarrow(0 \rightarrow x)^{\prime} \approx 0 \rightarrow x^{\prime}, \\
& \text { 9. } 0 \rightarrow(x \rightarrow y) \approx x \rightarrow(0 \rightarrow y), \\
& \text { 10. } 0 \rightarrow\left(x \rightarrow y^{\prime}\right)^{\prime} \approx 0 \rightarrow\left(x^{\prime} \rightarrow y\right), \\
& \text { 11. } x \rightarrow\left(y \rightarrow x^{\prime}\right) \approx y \rightarrow x^{\prime}, \\
& \text { 12. }(x \rightarrow y) \rightarrow(y \rightarrow x) \approx y \rightarrow x, \\
& \text { 13. }(x \rightarrow y) \rightarrow(y \rightarrow z) \approx\left(0 \rightarrow x^{\prime}\right) \rightarrow(y \rightarrow z), \\
& \text { 14. }(x \rightarrow y)^{\prime} \rightarrow y \approx x \rightarrow y, \\
& \text { 15. }(x \rightarrow y) \rightarrow((0 \rightarrow y) \rightarrow z) \approx(x \rightarrow y) \rightarrow z, \\
& \text { 16. }(x \rightarrow y) \rightarrow((z \rightarrow y) \rightarrow(u \rightarrow z)) \approx(x \rightarrow y) \rightarrow(u \rightarrow z) .
\end{aligned}
$$

Proof. For the proofs of items (1), (3), (4), (9), (10), (11) we refer the reader to [CS16a], and for the proofs of items $(2),(6),(7),(8)$ to [CS16b]. Items (5), (12) are proved in [CS16c]. For the proofs of items (13), (14) we refer the reader to [CS17]. Finally, for the proof of (15), the reader is referred to the proof of the equation (3.4) in the proof of Lemma 3.1 of [CS17].

Proof of (16): Let $a, b, c, d \in A$. Hence, $(a \rightarrow b) \rightarrow((c \rightarrow b) \rightarrow(d \rightarrow c))$ $\stackrel{(I)}{=}(a \rightarrow b) \rightarrow\left(\left((d \rightarrow c)^{\prime} \rightarrow c\right) \rightarrow(b \rightarrow(d \rightarrow c))^{\prime}\right)^{\prime} \stackrel{(14)}{=}(a \rightarrow b) \rightarrow$ $\left((d \rightarrow c) \rightarrow(b \rightarrow(d \rightarrow c))^{\prime}\right)^{\prime} \stackrel{(3)}{=}(a \rightarrow b) \rightarrow(((d \rightarrow c) \rightarrow b) \rightarrow(d \rightarrow c))$ $\stackrel{(4)}{=}(a \rightarrow b) \rightarrow((0 \rightarrow b) \rightarrow(d \rightarrow c)) \stackrel{(15)}{=}(a \rightarrow b) \rightarrow(d \rightarrow c)$, completing the proof.

LEMMA 2.5. Let $\mathbf{A} \in \mathcal{I}_{2,0}$ such that $\mathbf{A} \models 0 \approx 0^{\prime}$, then $\mathbf{A} \models 0 \rightarrow x \approx x$.

Proof. Let $a \in A$. Then $a=0^{\prime} \rightarrow a=(0 \rightarrow 0) \rightarrow a=\left(0^{\prime} \rightarrow 0\right) \rightarrow a \stackrel{2.2(4)}{=}$ $0 \rightarrow a$.

LEMMA 2.6. [CS18a] Let $\mathbf{A} \in \mathcal{I}_{2,0}$ such that $\mathbf{A} \models 0 \rightarrow x \approx x$, then $\mathbf{A} \models(x \rightarrow y)^{\prime} \approx x^{\prime} \rightarrow y^{\prime}$.

Throughout the rest of this paper, $\mathrm{A} \in \mathcal{S}$. 
LEMMA 2.7. [CS18a] A satisfies:

1. $x \rightarrow(y \rightarrow z) \approx y \rightarrow(x \rightarrow z)$,

2. $x^{\prime} \rightarrow y \approx y^{\prime} \rightarrow x$.

LEMMA 2.8. Let $\mathbf{A} \in \mathcal{S}$ such that $\mathbf{A} \models 0 \rightarrow x \approx x \rightarrow x$, then

1. $\mathbf{A} \models 0 \rightarrow(x \rightarrow x) \approx x \rightarrow x$.

2. $\mathbf{A} \models 0 \rightarrow x^{\prime} \approx 0 \rightarrow x$.

Proof. Let $a \in A$.

1. Observe that $0 \rightarrow(a \rightarrow a) \stackrel{\text { hyp }}{=}(a \rightarrow a) \rightarrow(a \rightarrow a) \stackrel{2.4(12)}{=} a \rightarrow a$.

2. $0 \rightarrow a \stackrel{h y p}{=} a \rightarrow a \stackrel{2.7(2)}{=} a^{\prime} \rightarrow a^{\prime} \stackrel{\text { hyp }}{=} 0 \rightarrow a^{\prime}$.

This proves the lemma.

LEMMA 2.9. Let $\mathbf{A} \in \mathcal{S}$ such that $\mathbf{A} \models 0 \rightarrow(x \rightarrow x) \approx x \rightarrow x$, then $\mathbf{A}$ satisfies the following identities:

1. $(x \rightarrow x) \rightarrow y^{\prime} \approx((x \rightarrow x) \rightarrow y)^{\prime}$,

2. $(x \rightarrow x) \rightarrow(y \rightarrow z) \approx((x \rightarrow x) \rightarrow y) \rightarrow z$,

3. $(x \rightarrow y) \rightarrow(x \rightarrow y) \approx(x \rightarrow x) \rightarrow(y \rightarrow y)$.

Proof. Items (1) and (2) follow from [CS18a, Lemma 3.4]. Let us prove (3). Let $a, b \in A$. Observe that

$(a \rightarrow a) \rightarrow\left(0 \rightarrow a^{\prime}\right) \stackrel{2.7(1)}{=} 0 \rightarrow\left((a \rightarrow a) \rightarrow a^{\prime}\right) \stackrel{2.4(1)}{=} 0 \rightarrow\left(\left(a \rightarrow 0^{\prime}\right) \rightarrow a^{\prime}\right)$ $\stackrel{2.3(2)}{=} 0 \rightarrow\left(\left(0 \rightarrow a^{\prime}\right) \rightarrow a^{\prime}\right) \stackrel{2.7(1)}{=}\left(0 \rightarrow a^{\prime}\right) \rightarrow\left(0 \rightarrow a^{\prime}\right) \stackrel{2.4(6) \stackrel{\text { and }(9)}{=}}{=} \rightarrow\left(a^{\prime} \rightarrow a^{\prime}\right)$ $\stackrel{\text { hyp }}{=} a^{\prime} \rightarrow a^{\prime} \stackrel{2.7(2)}{=} a \rightarrow a$. Hence,

$$
\mathbf{A} \models(x \rightarrow x) \rightarrow\left(0 \rightarrow x^{\prime}\right) \approx x \rightarrow x
$$

Since

$$
\begin{aligned}
& (a \rightarrow b) \rightarrow(a \rightarrow b) \stackrel{2.7(1)}{=} a \rightarrow((a \rightarrow b) \rightarrow b) \stackrel{2.7(2)}{=} a \rightarrow\left(b^{\prime} \rightarrow(a \rightarrow b)^{\prime}\right) \\
& \stackrel{2.7(1)}{=} b^{\prime} \rightarrow\left(a \rightarrow(a \rightarrow b)^{\prime}\right) \stackrel{2.7(2)}{=} b^{\prime} \rightarrow\left((a \rightarrow b) \rightarrow a^{\prime}\right) \stackrel{(I)}{=} b^{\prime} \rightarrow((a \rightarrow a) \rightarrow \\
& \left.\left(b \rightarrow a^{\prime}\right)^{\prime}\right)^{\prime} \stackrel{2.7(2)}{=} b^{\prime} \rightarrow\left((a \rightarrow a) \rightarrow\left(a \rightarrow b^{\prime}\right)^{\prime}\right)^{\prime} \stackrel{(1)}{=} b^{\prime} \rightarrow((a \rightarrow a) \rightarrow(a \rightarrow
\end{aligned}
$$




$$
\begin{aligned}
& \left.\left.b^{\prime}\right)\right)^{\prime \prime} \stackrel{x^{\prime \prime} \approx x}{=} b^{\prime} \rightarrow\left((a \rightarrow a) \rightarrow\left(a \rightarrow b^{\prime}\right)\right) \stackrel{2.7(1)}{=}(a \rightarrow a) \rightarrow\left(b^{\prime} \rightarrow\left(a \rightarrow b^{\prime}\right)\right) \\
& \stackrel{(2)}{=}\left((a \rightarrow a) \rightarrow b^{\prime}\right) \rightarrow\left(a \rightarrow b^{\prime}\right) \stackrel{2.7(2)}{=}\left((a \rightarrow a) \rightarrow b^{\prime}\right) \rightarrow\left(b \rightarrow a^{\prime}\right) \stackrel{2.7(1)}{=} b \rightarrow \\
& \left(\left((a \rightarrow a) \rightarrow b^{\prime}\right) \rightarrow a^{\prime}\right) \stackrel{(2)}{=} b \rightarrow\left((a \rightarrow a) \rightarrow\left(b^{\prime} \rightarrow a^{\prime}\right)\right) \stackrel{2.7(2)}{=} b \rightarrow((a \rightarrow a) \rightarrow \\
& (a \rightarrow b)) \stackrel{2.7(1)}{=}(a \rightarrow a) \rightarrow(b \rightarrow(a \rightarrow b)) \stackrel{2.7(1)}{=}(a \rightarrow a) \rightarrow(a \rightarrow(b \rightarrow b)) \\
& \left.\stackrel{2.7(2)}{=}(a \rightarrow a) \rightarrow\left((b \rightarrow b)^{\prime} \rightarrow a^{\prime}\right)=(a \rightarrow a) \rightarrow((b \rightarrow b) \rightarrow 0) \rightarrow a^{\prime}\right) \\
& \stackrel{(2)}{=}(a \rightarrow a) \rightarrow\left((b \rightarrow b) \rightarrow\left(0 \rightarrow a^{\prime}\right)\right) \stackrel{2.7(1)}{=}(b \rightarrow b) \rightarrow\left((a \rightarrow a) \rightarrow\left(0 \rightarrow a^{\prime}\right)\right) \\
& \stackrel{(2.1)}{=}(b \rightarrow b) \rightarrow(a \rightarrow a), \text { we have }
\end{aligned}
$$

$$
\mathbf{A}=(x \rightarrow y) \rightarrow(x \rightarrow y) \approx(y \rightarrow y) \rightarrow(x \rightarrow x) .
$$

Consequently,

$$
\begin{aligned}
& (a \rightarrow b) \rightarrow(a \rightarrow b) \stackrel{2.7(2)}{=}\left(b^{\prime} \rightarrow a^{\prime}\right) \rightarrow\left(b^{\prime} \rightarrow a^{\prime}\right) \stackrel{(2.2)}{=}\left(a^{\prime} \rightarrow a^{\prime}\right) \rightarrow\left(b^{\prime} \rightarrow b^{\prime}\right) \\
& \stackrel{2.7(2) .}{=}(a \rightarrow a) \rightarrow(b \rightarrow b) .
\end{aligned}
$$

LEMMA 2.10. [CS18a] Let $\mathbf{A} \models x \rightarrow x \approx x$. Then $\mathbf{A} \models x^{\prime} \approx x$.

LEMMA 2.11. Let $\mathbf{A}=0 \rightarrow x \approx x$. Then $\mathbf{A} \models x \rightarrow(x \rightarrow x) \approx(x \rightarrow$ $x) \rightarrow x$.

Proof. By Lemma 2.6,

$$
\mathbf{A} \models(x \rightarrow y)^{\prime} \approx x^{\prime} \rightarrow y^{\prime}
$$

Let $a \in A$. Then $(a \rightarrow a) \rightarrow a \stackrel{2.7(2)}{=} a^{\prime} \rightarrow(a \rightarrow a)^{\prime} \stackrel{(2.3)}{=} a^{\prime} \rightarrow\left(a^{\prime} \rightarrow a^{\prime}\right)$ $\stackrel{2.4(7)}{=} a^{\prime} \rightarrow a^{\prime} \stackrel{2.7(2)}{=} a \rightarrow a \stackrel{2.4(7)}{=} a \rightarrow(a \rightarrow a)$.

Let $E$ be the set consisting of the terms:

$$
\begin{aligned}
& t_{1}(x, y, z, t)=((x \rightarrow y) \rightarrow z) \rightarrow t, \\
& t_{2}(x, y, z, t)=z \rightarrow((y \rightarrow x) \rightarrow t), \\
& t_{3}(x, y, z, t)=(y \rightarrow x) \rightarrow(z \rightarrow t), \\
& t_{4}(x, y, z, t)=((z \rightarrow y) \rightarrow x) \rightarrow t, \text { and } \\
& t_{5}(x, y, z, t)=(y \rightarrow z) \rightarrow(x \rightarrow t) .
\end{aligned}
$$

LEMMA 2.12. If $\mathbf{A} \models 0 \rightarrow x \approx x$, then $\mathbf{A} \models e_{1} \approx e_{2}$ where $e_{1}, e_{2} \in E$.

Proof. Since $\mathbf{A} \models 0 \rightarrow x \approx x$, by Lemma 2.6,

$$
\mathbf{A} \models(x \rightarrow y)^{\prime} \approx x^{\prime} \rightarrow y^{\prime}
$$


Let $a, b, c, d \in A$. Then

$((a \rightarrow b) \rightarrow c) \rightarrow d \stackrel{2.7(2)}{=} d^{\prime} \rightarrow((a \rightarrow b) \rightarrow c)^{\prime} \stackrel{(2.4)}{=}(d \rightarrow((a \rightarrow b) \rightarrow c))^{\prime} \stackrel{2.7(2)}{=}$
$\left(d \rightarrow\left(c^{\prime} \rightarrow(a \rightarrow b)^{\prime}\right)\right)^{\prime} \stackrel{2.7(1)}{=}\left(c^{\prime} \rightarrow\left(d \rightarrow(a \rightarrow b)^{\prime}\right)\right)^{\prime} \stackrel{(2.4)}{=} c^{\prime \prime} \rightarrow\left(d \rightarrow(a \rightarrow b)^{\prime}\right)^{\prime}$
$\stackrel{x_{x^{\prime \prime}}}{=} c \rightarrow\left(d \rightarrow(a \rightarrow b)^{\prime}\right)^{\prime} \stackrel{2.7(2)}{=} c \rightarrow\left(d \rightarrow\left(b^{\prime} \rightarrow a^{\prime}\right)^{\prime}\right)^{\prime} \stackrel{(2.4)}{=} c \rightarrow(d \rightarrow(b \rightarrow a))^{\prime}$
$\stackrel{(2.4)}{=} c \rightarrow\left(d^{\prime} \rightarrow(b \rightarrow a)^{\prime}\right) \stackrel{2.7(2)}{=} c \rightarrow((b \rightarrow a) \rightarrow d) \stackrel{2.7(1)}{=}(b \rightarrow a) \rightarrow(c \rightarrow d)$, proving $t_{1} \approx t_{2}$ and $t_{1} \approx t_{3}$.

Next, $((a \rightarrow b) \rightarrow c) \rightarrow d \stackrel{2.7(2)}{=}\left(c^{\prime} \rightarrow(a \rightarrow b)^{\prime}\right) \rightarrow d \stackrel{(2.4)}{=}\left(c^{\prime} \rightarrow\left(a^{\prime} \rightarrow\right.\right.$ $\left.\left.b^{\prime}\right)\right) \rightarrow d \stackrel{2.7(1)}{=}\left(a^{\prime} \rightarrow\left(c^{\prime} \rightarrow b^{\prime}\right)\right) \rightarrow d \stackrel{2.7(2)}{=}\left(\left(c^{\prime} \rightarrow b^{\prime}\right)^{\prime} \rightarrow a\right) \rightarrow d \stackrel{(2.4)}{=}((c \rightarrow$ b) $\rightarrow a) \rightarrow d$, proving $t_{1} \approx t_{4}$.

Also, we have that

$c \rightarrow((b \rightarrow a) \rightarrow d) \stackrel{2.7(2)}{=} c \rightarrow\left(d^{\prime} \rightarrow(b \rightarrow a)^{\prime}\right) \stackrel{2.7(2)}{=} c \rightarrow\left(d^{\prime} \rightarrow\left(a^{\prime} \rightarrow b^{\prime}\right)^{\prime}\right)$ $\stackrel{(2.4)}{=} c \rightarrow\left(d^{\prime} \rightarrow(a \rightarrow b)\right) \stackrel{2.7(1)}{=} c \rightarrow\left(a \rightarrow\left(d^{\prime} \rightarrow b\right)\right) \stackrel{2.7(1)}{=} a \rightarrow\left(c \rightarrow\left(d^{\prime} \rightarrow b\right)\right)$ $\stackrel{2.7(1)}{=} a \rightarrow\left(d^{\prime} \rightarrow(c \rightarrow b)\right) \stackrel{2.7(2)}{=} a \rightarrow\left((c \rightarrow b)^{\prime} \rightarrow d\right) \stackrel{2.7(2)}{=} a \rightarrow\left(\left(b^{\prime} \rightarrow c^{\prime}\right)^{\prime} \rightarrow d\right)$ $\stackrel{(2.4)}{=} a \rightarrow((b \rightarrow c) \rightarrow d) \stackrel{2.7(1),}{=}(b \rightarrow c) \rightarrow(a \rightarrow d)$, proving $t_{2} \approx t_{5}$.

LEMMA 2.13. If $\mathbf{A} \models 0 \rightarrow x \approx x$, then $\mathbf{A} \models x \rightarrow((x \rightarrow x) \rightarrow y) \approx(x \rightarrow$ $(x \rightarrow x)) \rightarrow y$.

Proof. By Lemma 2.12, $\mathbf{A} \models e_{1} \approx e_{2}$ for all $e_{1}, e_{2} \in E$. Consider $a, b \in A$. We have that $a \rightarrow((a \rightarrow a) \rightarrow b)=t_{2}(a, a, a, b)=t_{4}(a, a, a, b)=((a \rightarrow$ $a) \rightarrow a) \rightarrow b \stackrel{2.11}{=}(a \rightarrow(a \rightarrow a)) \rightarrow b$, proving the lemma.

\section{LEMMA 2.14. A satisfies:}

1. $(x \rightarrow x) \rightarrow(x \rightarrow x) \approx x \rightarrow(x \rightarrow(x \rightarrow x))$,

2. $((x \rightarrow x) \rightarrow x) \rightarrow x \approx x \rightarrow(x \rightarrow(x \rightarrow x))$,

3. $(x \rightarrow y) \rightarrow(y \rightarrow z) \approx((y \rightarrow x) \rightarrow y) \rightarrow z$,

4. $y \rightarrow((x \rightarrow y) \rightarrow z) \approx((y \rightarrow x) \rightarrow y) \rightarrow z$,

5. $(x \rightarrow x) \rightarrow(x \rightarrow y) \approx x \rightarrow((x \rightarrow x) \rightarrow y)$,

6. $x \rightarrow((x \rightarrow x) \rightarrow y) \approx((x \rightarrow x) \rightarrow x) \rightarrow y$,

7. $x \rightarrow((y \rightarrow x) \rightarrow x) \approx((x \rightarrow y) \rightarrow x) \rightarrow x$,

8. $x \rightarrow((y \rightarrow x) \rightarrow y) \approx((x \rightarrow y) \rightarrow x) \rightarrow y$. 
Proof. Let $a, b, c \in A$.

1. Observe that

$$
\begin{aligned}
& (a \rightarrow a) \rightarrow(a \rightarrow a) \stackrel{2.7(1)}{=} a \rightarrow((a \rightarrow a) \rightarrow a) \stackrel{2.4(4)}{=} a \rightarrow((0 \rightarrow a) \rightarrow a) \\
& \stackrel{2.7(1)}{=}(0 \rightarrow a) \rightarrow(a \rightarrow a) \stackrel{2.4(5)}{=} a \rightarrow(a \rightarrow a) \stackrel{2.4(7)}{=} a \rightarrow(a \rightarrow(a \rightarrow a)) .
\end{aligned}
$$

2. $((a \rightarrow a) \rightarrow a) \rightarrow a \stackrel{2.4(4)}{=}((0 \rightarrow a) \rightarrow a) \rightarrow a \stackrel{2.4(2)}{=} a \rightarrow a \stackrel{2.4(7)}{=} a \rightarrow$ $(a \rightarrow a) \stackrel{2.4(7)}{=} a \rightarrow(a \rightarrow(a \rightarrow a))$.

3. $(a \rightarrow b) \rightarrow(b \rightarrow c) \stackrel{2.4(13)}{=}\left(0 \rightarrow a^{\prime}\right) \rightarrow(b \rightarrow c) \stackrel{(I)}{=}\left(\left((b \rightarrow c)^{\prime} \rightarrow\right.\right.$ $\left.0) \rightarrow\left(a^{\prime} \rightarrow(b \rightarrow c)\right)^{\prime}\right)^{\prime} \stackrel{x \approx x^{\prime \prime}}{=}\left((b \rightarrow c) \rightarrow\left(a^{\prime} \rightarrow(b \rightarrow c)\right)^{\prime}\right)^{\prime} \stackrel{2.7(2)}{=}$ $\left(\left(a^{\prime} \rightarrow(b \rightarrow c)\right) \rightarrow(b \rightarrow c)^{\prime}\right)^{\prime} \stackrel{2.7(1)}{=}\left(\left(b \rightarrow\left(a^{\prime} \rightarrow c\right)\right) \rightarrow(b \rightarrow c)^{\prime}\right)^{\prime}$ $\stackrel{2.7(2)}{=}\left(\left(b \rightarrow\left(c^{\prime} \rightarrow a\right)\right) \rightarrow(b \rightarrow c)^{\prime}\right)^{\prime} \stackrel{2.7(1)}{=}\left(\left(c^{\prime} \rightarrow(b \rightarrow a)\right) \rightarrow(b \rightarrow c)^{\prime}\right)^{\prime}$ $\stackrel{(I)}{=}((b \rightarrow a) \rightarrow b) \rightarrow c$.

4. $b \rightarrow((a \rightarrow b) \rightarrow c) \stackrel{2.7(1)}{=}(a \rightarrow b) \rightarrow(b \rightarrow c) \stackrel{(3)}{=}((b \rightarrow a) \rightarrow b) \rightarrow c$.

For (5) use Lemma 2.7 (1), and for (6) use Lemma 2.7 (1) and item (3). (7) is a special case of (4). Finally, one can use Lemma 2.7 (1) and item (3) to prove (8).

\section{Weak associative laws of length 3}

In this section we examine all the weak associative laws of length 3.

\subsection{With one variable:}

The only word of length 3 with 1 variable is:

$\mathrm{A}:\langle x, x, x\rangle$.

Ways in which the word A can be bracketed (where $a$ is just a place holder) are:

$1: a \rightarrow(a \rightarrow a), \quad 2:(a \rightarrow a) \rightarrow a$.

The only weak associative identitiy in this category is:

1: $(31 \mathrm{~A} 12) x \rightarrow(x \rightarrow x) \approx(x \rightarrow x) \rightarrow x$. 


\subsection{With 2 variables:}

Possible words of length 3 with 2 variables are:
$\mathrm{A}:\langle x, x, y\rangle$,
$\mathrm{B}:\langle x, y, x\rangle$,
$\mathrm{C}:\langle x, y, y\rangle$.

Ways in which a word of length 3 can be bracketed:
$1: a \rightarrow(a \rightarrow a)$,
2: $(a \rightarrow a) \rightarrow a$.

The weak associative identities in this category are:

1: (LALT) $x \rightarrow(x \rightarrow y) \approx(x \rightarrow x) \rightarrow y$ (the left-alternative law)

$2:($ FLEX) $x \rightarrow(y \rightarrow x) \approx(x \rightarrow y) \rightarrow x$ (the flexible law)

3: $(\mathrm{RALT}) x \rightarrow(y \rightarrow y) \approx(x \rightarrow y) \rightarrow y$ (the right-alternative law)

We should note that we did not follow our convention in this case, since these identities are well known by the above names. We let $\mathcal{L} \mathcal{A} \mathcal{L} \mathcal{T}, \mathcal{F} \mathcal{L E X}$ and $\mathcal{R} \mathcal{A} \mathcal{L} \mathcal{T}$ denote, respectively, the subvarieties $\mathcal{S}$ defined by (LALT), (FLEX) and (RALT).

THEOREM 3.1. $\mathcal{F} \mathcal{L} \mathcal{E} \mathcal{X} \mathcal{R} \mathcal{A L} \mathcal{T}=\mathcal{L} \mathcal{A L} \mathcal{T}=\mathcal{S} \mathcal{L}$.

Proof. Let $\mathrm{A} \in \mathcal{L} \mathcal{A} \mathcal{L} \mathcal{T} \cup \mathcal{F} \mathcal{L} \mathcal{E} \cup \mathcal{R} \mathcal{A} \mathcal{L} \mathcal{T}$ and $a \in A$.

First, let $\mathbf{A} \in \mathcal{L} \mathcal{A L T}$. Then $a \stackrel{x \approx x^{\prime \prime}}{=} a^{\prime \prime} \stackrel{2.2(4)}{=}\left(a^{\prime \prime} \rightarrow a^{\prime}\right)^{\prime} \stackrel{x \approx x^{\prime \prime}}{=}\left(a \rightarrow a^{\prime}\right)^{\prime}$ $=(a \rightarrow(a \rightarrow 0))^{\prime} \stackrel{(L A L T)}{=}((a \rightarrow a) \rightarrow 0)^{\prime}=(a \rightarrow a)^{\prime \prime} \stackrel{x \approx x^{\prime \prime}}{=} a \rightarrow a$.

Next, let $\mathbf{A} \in \mathcal{F} \mathcal{L E X}$. Then $a \stackrel{2.2(4)}{=} a^{\prime} \rightarrow a \stackrel{2.2(4)}{=}\left(a^{\prime \prime} \rightarrow a^{\prime}\right) \rightarrow a \stackrel{x \approx x^{\prime \prime}}{=}(a \rightarrow$ $\left.a^{\prime}\right) \rightarrow a \stackrel{(F L E X)}{=} a \rightarrow\left(a^{\prime} \rightarrow a\right) \stackrel{2.2(4)}{=} a \rightarrow a$.

Finally, let $\mathbf{A} \in \mathcal{R} \mathcal{A} \mathcal{L} \mathcal{T}$. Then $a \rightarrow a \stackrel{x \approx x^{\prime \prime}}{=} a^{\prime \prime} \rightarrow a=((a \rightarrow 0) \rightarrow 0) \rightarrow a$ $\stackrel{(R A L T)}{=}(a \rightarrow(0 \rightarrow 0)) \rightarrow a=\left(a \rightarrow 0^{\prime}\right) \rightarrow a \stackrel{2.4(1)}{=}\left(a \rightarrow a^{\prime}\right) \rightarrow a \stackrel{x \approx x^{\prime \prime}}{=}\left(a^{\prime \prime} \rightarrow\right.$ $\left.a^{\prime}\right) \rightarrow a \stackrel{2.2(4)}{=} a^{\prime} \rightarrow a \stackrel{2.2(4)}{=} a$. Therefore, $\mathbf{A} \models x \rightarrow x \approx x$. By Lemma 2.10, $\mathbf{A} \equiv x \approx x^{\prime}$ and consequently, $\mathbf{A} \in \mathcal{S} \mathcal{L}$.

For the converse, it is easy to verify that $\mathbf{2}_{\mathbf{s}}$ satisfies (FLEX), (RALT) and (LALT). The theorem follows since $\mathbf{V}\left(\mathbf{2}_{\mathbf{s}}\right)=\mathcal{S} \mathcal{L}$.

\subsection{With 3 variables:}

The only word of length 3 with 3 variables is:

$\mathrm{A}:\langle x, y, z\rangle$. 
Ways in which a word of length 3 can be bracketed:

$1: a \rightarrow(a \rightarrow a), \quad 2:(a \rightarrow a) \rightarrow a$.

The only weak associative identitiy in this category is:

$(33 \mathcal{A} 12) x \rightarrow(y \rightarrow z) \approx(x \rightarrow y) \rightarrow z$ (associative law).

LEMMA 3.2. $33 \mathcal{A} 12=\mathcal{S} \mathcal{L}$.

Proof. By [CS16a], $\mathcal{S} \mathcal{L} \subseteq 33 \mathcal{A} 12$. Hence let us consider $\mathbf{A} \in 33 \mathcal{A} 12$ and $a \in A$. Observe that $0^{\prime} \stackrel{2.8(2)}{=} 0 \rightarrow 0^{\prime}=0 \rightarrow(0 \rightarrow 0)=(0 \rightarrow 0) \rightarrow 0=0^{\prime} \rightarrow$ $0 \stackrel{2.2(1)}{=} 0$. Then

$$
\mathbf{A} \models 0^{\prime} \approx 0
$$

Therefore, $a \stackrel{2.2(4)}{=} a^{\prime} \rightarrow a=(a \rightarrow 0) \rightarrow a \stackrel{(33 A 12)}{=} a \rightarrow(0 \rightarrow a) \stackrel{(3.1)}{=} a \rightarrow\left(0^{\prime} \rightarrow\right.$ a) $\stackrel{2.2(1)}{=} a \rightarrow a$. Consequently, $\mathbf{A} \in \mathcal{S} \mathcal{L}$ by Lemmas 2.1 and 2.10.

\section{Weak associative laws with length 4 and with 1 variable.}

The only word of length 4 with 1 variable is:

$\mathrm{A}:\langle x, x, x, x\rangle$.

Ways in which a word of length 4 can be bracketed are:

$1: a \rightarrow(a \rightarrow(a \rightarrow a)), \quad 3:(a \rightarrow a) \rightarrow(a \rightarrow a), \quad 5:((a \rightarrow a) \rightarrow a) \rightarrow a$.

$2: a \rightarrow((a \rightarrow a) \rightarrow a), \quad 4:(a \rightarrow(a \rightarrow a)) \rightarrow a$,

By now, we believe that the reader is well acquainted with our notation for identities. So, we will, no longer, present the list of the identities in this and the remaining categories.

LEMMA 4.1. The following hold:

1. $41 \mathcal{A} 12=41 \mathcal{A} 13$,

2. $41 \mathcal{A} 24=41 \mathcal{A} 34$,

3. $41 \mathcal{A} 25=41 \mathcal{A} 35$,

4. $41 \mathcal{A} 23=\mathcal{S}$, 
5. $41 \mathcal{A} 13=41 \mathcal{A} 15=41 \mathcal{A} 35=\mathcal{S}$,

6. $41 \mathcal{A} 14=41 \mathcal{A} 34=41 \mathcal{A} 45$.

Proof. Items (1), (2), (3) and (4) follow from Lemma 2.7 (1). For (5), observe that $\mathcal{S} \subseteq 41 \mathcal{A} 13$ by Lemma $2.14(1), \mathcal{S} \subseteq 41 \mathcal{A} 15$ by Lemma 2.14 (2), and $\mathcal{S} \subseteq 41 \mathcal{A} 35$ by Lemma $2.14(6)$ and (5).

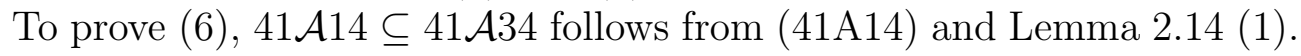
From (41A34), Lemma 2.7 (1) and Lemma 2.14 (6) we have that $41 \mathcal{A} 34 \subseteq$ $41 \mathcal{A} 45$. The inclusion $41 \mathcal{A} 45 \subseteq 41 \mathcal{A} 14$ follows from (41A45) and Lemma $2.14(2)$.

LEMMA 4.2. $41 \mathcal{A} 14=31 \mathcal{A} 12$.

Proof. In view of Lemma $2.4(7)$, we get $31 \mathcal{A} 12 \subseteq 41 \mathcal{A} 14$. So, we will prove the converse. Let $\mathbf{A} \in 41 \mathcal{A} 14$. Hence $0 \stackrel{2.2(1)}{=} 0^{\prime} \rightarrow 0 \stackrel{2.2(4)}{=}\left(0^{\prime \prime} \rightarrow 0^{\prime}\right) \rightarrow 0$ $\stackrel{\left(I_{0}\right)}{=}\left(0 \rightarrow 0^{\prime}\right) \rightarrow 0=(0 \rightarrow(0 \rightarrow 0)) \rightarrow 0 \stackrel{(41 A 14)}{=} 0 \rightarrow(0 \rightarrow(0 \rightarrow 0)) \stackrel{2.4(7)}{=} 0 \rightarrow 0$ $=0^{\prime}$. Therefore

$$
\mathbf{A} \models 0 \approx 0^{\prime}
$$

Let $a \in A$. Then we have that $(a \rightarrow a) \rightarrow a \stackrel{2.4(4)}{=}(0 \rightarrow a) \rightarrow a \stackrel{(4.1)}{=}\left(0^{\prime} \rightarrow\right.$ $a) \rightarrow a \stackrel{2.2(1)}{=} a \rightarrow a \stackrel{2.4(7)}{=} a \rightarrow(a \rightarrow a)$. Thus $41 \mathcal{A} 14 \subseteq 31 \mathcal{A} 12$, completing the proof.

\section{Weak associative laws with length 4 and with 2 variables.}

Possible words of length 4 with 2 variables are:
$\mathrm{A}:\langle x, x, x, y\rangle$
$\mathrm{C}:\langle x, x, y, y\rangle$,
$\mathrm{E}:\langle x, y, x, y\rangle$,
$\mathrm{B}:\langle x, x, y, x\rangle$,
$\mathrm{D}:\langle x, y, x, x\rangle$,
$\mathrm{F}:\langle x, y, y, x\rangle$,
$\mathrm{G}:\langle x, y, y, y\rangle$.

Ways in which a word of length 4 can be bracketed are:

1: $a \rightarrow(a \rightarrow(a \rightarrow a))$,

4: $(a \rightarrow(a \rightarrow a)) \rightarrow a$,

2: $a \rightarrow((a \rightarrow a) \rightarrow a)$,

$5:((a \rightarrow a) \rightarrow a) \rightarrow a$.

$3:(a \rightarrow a) \rightarrow(a \rightarrow a)$,

It is easy to see that there are 70 identities in this category, and accordingly there are 70 subvarieties of $\mathcal{S}$ defined by them. As not all of them 
will be distinct, we will partition these 70 varieties subject to the relation of "being equal". We will choose one representative from each block of the partition. As will be shown below, some of them will equal $\mathcal{S} \mathcal{L}$ and some others will equal $\mathcal{S}$. We will use $\mathcal{S} \mathcal{L}$ and $\mathcal{S}$ as representatives of the blocks whose varieties are equal to $\mathcal{S} \mathcal{L}$ and $\mathcal{S}$, respectively.

LEMMA 5.1. $\mathcal{S} \mathcal{L} \subseteq 42 \mathcal{X} i j$, for $\mathcal{X} \in\{A, B, C, D, E, F, G\}$ and for all $i, j$ such that $1 \leq i<j \leq 5$.

Proof. By a routine computation, it is easy to check that $\mathbf{2}_{\mathbf{s}} \in 42 \mathcal{X} i j$ for all $1 \leq i<j \leq 5$. Then the proof is complete since $\mathcal{V}\left(\mathbf{2}_{\mathbf{s}}\right)=\mathcal{S} \mathcal{L}$, in view of [CS16a, Corollary 10.4].

LEMMA 5.2. We have

1. $42 \mathcal{A} 23=42 \mathcal{A} 25=42 \mathcal{A} 35=42 \mathcal{D} 25=42 \mathcal{E} 25=\mathcal{S}$,

2. $42 \mathcal{A} 12=42 \mathcal{A} 13=42 \mathcal{A} 15$ and $42 \mathcal{A} 24=42 \mathcal{A} 34$,

3. $42 \mathcal{D} 12=42 \mathcal{D} 15$ and $42 \mathcal{E} 12=42 \mathcal{E} 15$.

Proof. 1. From Lemma 2.14 (5) and (6) we have that

$$
42 \mathcal{A} 23=42 \mathcal{A} 25=42 \mathcal{A} 35=\mathcal{S} .
$$

Using Lemma 2.14 (7) and (8) we can conclude that

$$
42 \mathcal{D} 25=42 \mathcal{E} 25=\mathcal{S} .
$$

2. Follows from Lemma 2.14 (5) and (6).

3. Follows from Lemma 2.14 (7) and (8).

The proof is complete.

Let $M$ be the set consisting of the following pairs of varieties: $(42 \mathcal{B} 12,42 \mathcal{D} 13)$, $(42 \mathcal{B} 13,42 \mathcal{G} 12),(42 \mathcal{C} 12,42 \mathcal{E} 13),(42 \mathcal{C} 13,42 \mathcal{F} 12),(42 \mathcal{D} 12,42 \mathcal{G} 13),(42 \mathcal{D} 14,42 \mathcal{G} 14)$,

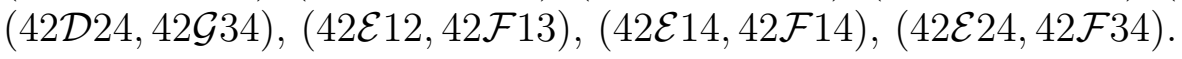

LEMMA 5.3. If $(\mathcal{A}, \mathcal{B}) \in M$ then $\mathcal{A}=\mathcal{B}$.

Proof. Follows from Lemma 2.7 (1). Rename the variables, if necessary. 
Let $N$ be the set consisting of the following varieties $42 \mathcal{A} 14,42 \mathcal{B} 14$, $42 \mathcal{B} 24,42 \mathcal{B} 34,42 \mathcal{B} 45,42 \mathcal{C} 14,42 \mathcal{C} 24,42 \mathcal{C} 34,42 \mathcal{C} 45,42 \mathcal{D} 14,42 \mathcal{D} 34,42 \mathcal{E} 12$, $42 \mathcal{E} 14,42 \mathcal{E} 23,42 \mathcal{E} 24,42 \mathcal{E} 34,42 \mathcal{E} 35,42 \mathcal{E} 45,42 \mathcal{F} 15,42 \mathcal{F} 23,42 \mathcal{F} 24,42 \mathcal{F} 45$, $42 \mathcal{G} 23,42 \mathcal{G} 24,42 \mathcal{G} 35,42 \mathcal{G} 45$.

THEOREM 5.4. If $\mathcal{X} \in N$ then $\mathcal{X}=\mathcal{S L}$.

Proof. In the proof below the following list of statements will be useful.

The identity $\left(I_{2,0}\right)$, Lemma $2.2(1)$, Lemma 2.2 (4), Lemma 2.4 (1),

(*) Lemma 2.4 (4), Lemma 2.4 (7), Lemma 2.4 (10), Lemma 2.7 (1) and Lemma 2.7 (2)

Let $\mathcal{X} \in N$. In view of Lemma 5.1, it suffices to prove that $\mathcal{X} \subseteq \mathcal{S L}$. In fact, by Lemma 2.1, it suffices to prove that $\mathcal{X}=x^{\prime} \approx x$.

Let $\mathbf{A} \in \mathcal{X}$ and let $a \in A$.

To facilitate a uniform presentation (and to make the proof shorter), we introduce the following notation, where $x_{0}, y_{0} \in \mathbf{A}$ :

The notation

$$
\mathcal{X} / x_{0}, y_{0}
$$

denotes the following statement:

"In the identity $(\mathrm{X})$ that defines the variety $\mathcal{X}$, relative to $\mathcal{S}$, if we assign $x:=x_{0}, y:=y_{0}$ (and simplify it using the list $(*)$ ), then $\mathbf{A} \models x \rightarrow x \approx x$ ".

Firstly, we consider the varieties associated with the following statements:

1. $42 \mathcal{A} 14 / a, 0$,

2. $42 \mathcal{B} 14 / a, a^{\prime}$,

3. $42 \mathcal{B} 24 / a, a^{\prime}$,

4. $42 \mathcal{C} 24 / a, 0$,

5. $42 \mathcal{C} 45 / a, 0$,
6. $42 \mathcal{E} 12 / a^{\prime}, a$,

7. $42 \mathcal{E} 23 / a^{\prime}, 0$,

8. $42 \mathcal{E} 35 / a^{\prime}, 0$,

9. $42 \mathcal{F} 24 / a, 0$,

10. $42 \mathcal{F} 45 / a, 0$.

We prove (7) as an illustration.

$a \stackrel{2.2(4)}{=} a^{\prime} \rightarrow a \stackrel{2.2(4)}{=}\left(a^{\prime \prime} \rightarrow a^{\prime}\right) \rightarrow a \stackrel{x \approx x^{\prime \prime}}{=}\left(a \rightarrow a^{\prime}\right) \rightarrow a \stackrel{2.4(4)}{=}\left(0 \rightarrow a^{\prime}\right) \rightarrow a$ $\stackrel{2.7(2)}{=} a^{\prime} \rightarrow\left(0 \rightarrow a^{\prime}\right)^{\prime}=a^{\prime} \rightarrow\left(\left(0 \rightarrow a^{\prime}\right) \rightarrow 0\right) \stackrel{(42 E 23)}{=}\left(a^{\prime} \rightarrow 0\right) \rightarrow\left(a^{\prime} \rightarrow 0\right)$ $=a^{\prime \prime} \rightarrow a^{\prime \prime} \stackrel{x \approx x^{\prime \prime}}{=} a \rightarrow a$. 
Hence the statement (7) is true. Similarly, one can verify the rest of the above statements is true, from which it follows that, in each of the above cases, $\mathcal{X} \models x \rightarrow x \approx x$.

Then, applying Lemma 2.10, we get that $\mathcal{X} \models x^{\prime} \approx x$.

The notation, where $x_{0}, y_{0}, x_{1}, y_{1}, \in \mathbf{A}$,

$$
\mathcal{X} / x_{0}, y_{0} / x_{1}, y_{1} / p \approx q
$$

is an abbreviation for the following statement:

"In the identity $(\mathrm{X})$ that defines the variety $\mathcal{X}$, relative to $\mathcal{S}$, if we assign $x:=x_{0}, y:=y_{0}$, (and simplify $(\mathrm{X})$ using the appropriate lemmas from the list $(*)$ ), we obtain that $\mathbf{A} \models 0^{\prime} \approx 0$; and then we assign $x:=x_{1}, y:=y_{1}$ in the identity $(\mathrm{X})$ (and simplify it using $0^{\prime}=0$ and the list $(*)$ ), then $\mathbf{A} \models p \approx q$."

Secondly, consider the varieties associated with the following statements:

1. $42 \mathcal{B} 45 / 0,0 / a, a^{\prime} / x \rightarrow x \approx x, \quad$ 5. $42 \mathcal{E} 34 / 0,0 / 0, a / x \rightarrow x \approx x$,

2. $42 \mathcal{C} 34 / 0,0 / a, 0 / x \rightarrow x \approx x$,

3. $42 \mathcal{E} 14 / 0,0 / a, 0 / x \rightarrow x \approx x$

6. $42 \mathcal{E} 45 / 0,0 / a, 0 / x \rightarrow x \approx x$

4. $42 \mathcal{E} 24 / 0,0 / a, 0 / x \rightarrow x \approx x$,

7. $42 \mathcal{F} 23 / 0,0^{\prime} / a, 0 / x \rightarrow x \approx x$.

As a sample, we prove (2) below:

$0 \stackrel{2.2(4)}{=} 0^{\prime} \rightarrow 0 \stackrel{2.2(4)}{=}\left(0^{\prime \prime} \rightarrow 0^{\prime}\right) \rightarrow 0=\left(0 \rightarrow 0^{\prime}\right) \rightarrow 0=(0 \rightarrow(0 \rightarrow 0)) \rightarrow 0$ $\stackrel{(42 C 34)}{=}(0 \rightarrow 0) \rightarrow(0 \rightarrow 0)=0^{\prime} \rightarrow 0^{\prime} \stackrel{2.2(1)}{=} 0^{\prime}$ then $a \rightarrow a \stackrel{2.2(1)}{=} 0^{\prime} \rightarrow(a \rightarrow a)$ $\stackrel{0 \approx 0^{\prime}}{=} 0 \rightarrow(a \rightarrow a) \stackrel{2.7(2)}{=} 0 \rightarrow\left(a^{\prime} \rightarrow a^{\prime}\right) \stackrel{2.4(10)}{=} 0 \rightarrow(a \rightarrow a)^{\prime}=0 \rightarrow((a \rightarrow a) \rightarrow$ $0) \stackrel{2.7(1)}{=}(a \rightarrow a) \rightarrow(0 \rightarrow 0) \stackrel{(42 C 34)}{=}(a \rightarrow(a \rightarrow 0)) \rightarrow 0=\left(a \rightarrow a^{\prime}\right)^{\prime} \stackrel{2.2(4)}{=} a^{\prime \prime}$ $\stackrel{x \approx x^{\prime \prime}}{=} a$. Hence, (2) holds. Similarly, one can verify that each of the above statements is true. So, it follows that in each case $\mathcal{X} \models x \rightarrow x \approx x$. Then, applying Lemma 2.10, we get that $\mathcal{X}=x^{\prime} \approx x$.

Thirdly, consider the varieties associated with the following statements:
1. $42 \mathcal{C} 14 / 0,0 / a, 0 / x^{\prime} \approx x$,
3. $42 \mathcal{D} 34 / 0,0 / 0, a / x^{\prime} \approx x$,
2. $42 \mathcal{D} 14 / 0,0 / 0, a / x^{\prime} \approx x$,
4. $42 \mathcal{G} 23 / 0^{\prime}, 0 / a, 0 / x^{\prime} \approx x$, 

5. $42 \mathcal{G} 24 / 0,0 / a, 0 / x^{\prime} \approx x$,
7. $42 \mathcal{G} 45 / 0,0 / a, 0 / x^{\prime} \approx x$.
6. $42 \mathcal{G} 35 / 0^{\prime}, 0 / a, 0 / x^{\prime} \approx x$

It is easy to verify that the above statements are true. Hence, it follows in each of the above cases that $\mathcal{X} \models x^{\prime} \approx x$.

Thus, the varieties still left to consider are $42 \mathcal{B} 34$ and $42 \mathcal{F} 15$.

Let $\mathbf{A} \in 42 \mathcal{B} 34$ and let $a \in A$. Since $a \stackrel{x \approx x^{\prime \prime}}{=} a^{\prime \prime} \stackrel{2.2(1)}{=}\left(0^{\prime} \rightarrow a^{\prime}\right)^{\prime}=((0 \rightarrow$ $0) \rightarrow(a \rightarrow 0))^{\prime} \stackrel{(42 B 34)}{=}((0 \rightarrow(0 \rightarrow a)) \rightarrow 0)^{\prime} \stackrel{2.4(7)}{=}((0 \rightarrow a) \rightarrow 0)^{\prime} \stackrel{x \approx x^{\prime \prime}}{=} 0 \rightarrow a$, we have that

$$
\mathbf{A} \models x \approx 0 \rightarrow x
$$

Therefore, $a \rightarrow a \stackrel{(5.1)}{=}(0 \rightarrow a) \rightarrow a \stackrel{2.4(4)}{=}(a \rightarrow a) \rightarrow a \stackrel{2.2(1)}{=}(a \rightarrow a) \rightarrow$ $\left(0^{\prime} \rightarrow a\right) \stackrel{(42 B 34)}{=}\left(a \rightarrow\left(a \rightarrow 0^{\prime}\right)\right) \rightarrow a \stackrel{2.4(7)}{=}\left(a \rightarrow 0^{\prime}\right) \rightarrow a \stackrel{2.4(1)}{=}\left(a \rightarrow a^{\prime}\right) \rightarrow a$ $\stackrel{x \approx x^{\prime \prime}}{=}\left(a^{\prime \prime} \rightarrow a^{\prime}\right) \rightarrow a \stackrel{2.2(4)}{=} a^{\prime} \rightarrow a \stackrel{2.2(4)}{=} a$. Hence

$$
\mathbf{A} \models x \approx x \rightarrow x
$$

Then, applying Lemma 2.10, we get that $\mathbf{A}=x^{\prime} \approx x$.

Let $\mathbf{A} \in 42 \mathcal{F} 15$ and let $a \in A$. If we replace $x:=0$ and $y:=0^{\prime}$ we obtain that

$$
\mathbf{A} \models 0 \approx 0^{\prime}
$$

Since $a \stackrel{2.2(1)}{=} 0^{\prime} \rightarrow a \stackrel{(5.2)}{=} 0 \rightarrow a \stackrel{2.2(4)}{=} 0 \rightarrow\left(a^{\prime} \rightarrow a\right) \stackrel{x \approx x^{\prime \prime}}{=} 0 \rightarrow\left(a^{\prime} \rightarrow a^{\prime \prime}\right)$ $=0 \rightarrow\left(a^{\prime} \rightarrow\left(a^{\prime} \rightarrow 0\right)\right) \stackrel{(42 F 15)}{=}\left(\left(0 \rightarrow a^{\prime}\right) \rightarrow a^{\prime}\right) \rightarrow 0 \stackrel{(5.2)}{=}\left(\left(0^{\prime} \rightarrow a^{\prime}\right) \rightarrow a^{\prime}\right) \rightarrow 0$ $\stackrel{2.2(1)}{=}\left(a^{\prime} \rightarrow a^{\prime}\right) \rightarrow 0 \stackrel{2.7(2)}{=}(a \rightarrow a) \rightarrow 0$, the identity

$$
\mathbf{A} \models x \approx(x \rightarrow x)^{\prime}
$$

holds in A. Hence $a^{\prime} \stackrel{(5.3)}{=}\left(a^{\prime} \rightarrow a^{\prime}\right)^{\prime} \stackrel{2.7(2)}{=}(a \rightarrow a)^{\prime} \stackrel{(5.3)}{=} a$, proving the theorem.

Let $I$ be the set consisting of the following identities: (42A24), (42A45), (42B13), (42B15), (42B23), (42B25), (42D24), (42D45), (42F25) and (42G15).

LEMMA 5.5. Let $(x) \in I$. If $\mathbf{A} \models(x)$ then $A \models 0 \rightarrow x \approx x$. 
Proof. Let $a \in A$. We consider the following cases:

Case 1: $\mathbf{A} \models(42 A 24)$. Then $a \stackrel{2.2(1)}{=} 0^{\prime} \rightarrow a \stackrel{2.2(4)}{=}\left(0^{\prime \prime} \rightarrow 0^{\prime}\right) \rightarrow a=(0 \rightarrow$ $\left.0^{\prime}\right) \rightarrow a=(0 \rightarrow(0 \rightarrow 0)) \rightarrow a \stackrel{(42 A 24)}{=} 0 \rightarrow((0 \rightarrow 0) \rightarrow a)=0 \rightarrow\left(0^{\prime} \rightarrow a\right)$ $\stackrel{2.2(1)}{=} 0 \rightarrow a$.

Case 2: $\mathbf{A} \models(42 A 45)$. We have $a \stackrel{2.2(1)}{=} 0^{\prime} \rightarrow a \stackrel{2.2(4)}{=}\left(0^{\prime \prime} \rightarrow 0^{\prime}\right) \rightarrow a$ $=\left(0 \rightarrow 0^{\prime}\right) \rightarrow a=(0 \rightarrow(0 \rightarrow 0)) \rightarrow a \stackrel{(42 A 45)}{=}((0 \rightarrow 0) \rightarrow 0) \rightarrow a$ $=\left(0^{\prime} \rightarrow 0\right) \rightarrow a \stackrel{2.2(1)}{=} 0 \rightarrow a$.

Case 3: $\mathbf{A} \models(42 B 13)$. In this case, we get $a \stackrel{x \approx x^{\prime \prime}}{=} a^{\prime \prime}=a^{\prime} \rightarrow 0 \stackrel{2.2(1)}{=} 0^{\prime} \rightarrow$ $\left(a^{\prime} \rightarrow 0\right)=(0 \rightarrow 0) \rightarrow\left(a^{\prime} \rightarrow 0\right) \stackrel{(42 B 13)}{=} 0 \rightarrow\left(0 \rightarrow\left(a^{\prime} \rightarrow 0\right)\right) \stackrel{2.4(7)}{=} 0 \rightarrow\left(a^{\prime} \rightarrow\right.$ $0)=0 \rightarrow a^{\prime \prime} \stackrel{x \approx x^{\prime \prime}}{=} 0 \rightarrow a$.

Case 4: $\mathbf{A}=(42 B 15)$. Then $a \stackrel{x \approx x^{\prime \prime}}{=} a^{\prime \prime}=a^{\prime} \rightarrow 0 \stackrel{2.2(1)}{=}\left(0^{\prime} \rightarrow a^{\prime}\right) \rightarrow 0$ $=\left((0 \rightarrow 0) \rightarrow a^{\prime}\right) \rightarrow 0 \stackrel{(42 B 15)}{=} 0 \rightarrow\left(0 \rightarrow\left(a^{\prime} \rightarrow 0\right)\right) \stackrel{2.4(7)}{=} 0 \rightarrow\left(a^{\prime} \rightarrow 0\right)$ $=0 \rightarrow a^{\prime \prime} \stackrel{x \approx x^{\prime \prime}}{=} 0 \rightarrow a$.

Case 5: If $\mathbf{A} \models(42 B 23), a \stackrel{x \approx x^{\prime \prime}}{=} a^{\prime \prime}=a^{\prime} \rightarrow 0 \stackrel{2.2(1)}{=} 0^{\prime} \rightarrow\left(a^{\prime} \rightarrow 0\right)=(0 \rightarrow$ $0) \rightarrow\left(a^{\prime} \rightarrow 0\right) \stackrel{(42 B 23)}{=} 0 \rightarrow\left(\left(0 \rightarrow a^{\prime}\right) \rightarrow 0\right) \stackrel{2.4(8)}{=} 0 \rightarrow a^{\prime \prime} \stackrel{x \approx x^{\prime \prime}}{=} 0 \rightarrow a$.

Case 6: $\mathbf{A} \models(42 B 25)$. One has $a \stackrel{x \approx x^{\prime \prime}}{=} a^{\prime \prime}=a^{\prime} \rightarrow 0 \stackrel{2.2(1)}{=}\left(0^{\prime} \rightarrow a^{\prime}\right) \rightarrow 0$ $=\left((0 \rightarrow 0) \rightarrow a^{\prime}\right) \rightarrow 0 \stackrel{(42 B 25)}{=} 0 \rightarrow\left(\left(0 \rightarrow a^{\prime}\right) \rightarrow 0\right) \stackrel{2.4(8)}{=} 0 \rightarrow a^{\prime \prime} \stackrel{x \approx x^{\prime \prime}}{=} 0 \rightarrow a$.

Case $7: \mathbf{A} \models(42 D 24)$. Then, setting $x:=0$ and $y:=0$ in the identity (42D24), we obtain that $0^{\prime}=0$. Then apply Lemma 2.5 .

Case 8: $\mathbf{A}=(42 D 45)$, Then, set $x:=0$ and $y:=0$ in the identity (42D45) to obtain that $0^{\prime}=0$. Now, apply Lemma 2.5 .

Case 9: $\mathbf{A} \models(42 F 25)$. and we consider $x:=0$ and $y:=0^{\prime}$ in the identity $(42 F 25)$ we obtain that $0^{\prime}=0$. Then apply Lemma 2.5 .

Case 10: $\mathbf{A} \models(42 G 15)$. Then, setting $x:=0^{\prime}$ and $y:=0$ in the identity $(42 G 15)$, it is easy to obtain that $0^{\prime}=0$. Then apply Lemma 2.5.

THEOREM 5.6. If $\mathbf{A} \models 0 \rightarrow x \approx x$, then $\mathbf{A} \models(y)$ for all $(y) \in I$.

Proof. Observe that, by Lemma 2.12,

$$
\mathbf{A} \models t_{1} \approx t_{2} \approx t_{4} \approx t_{5}
$$

Also, by Lemma 2.13, $\mathbf{A} \equiv(42 A 24)$. Hence, by Lemma 2.14 (6), $\mathbf{A}=$ $(42 A 45)$. Now, $\mathbf{A} \models(42 B 13)$ is true, since $(a \rightarrow a) \rightarrow(b \rightarrow a) \stackrel{2.7(1)}{=} b \rightarrow$ 
$((a \rightarrow a) \rightarrow a) \stackrel{2.11}{=} b \rightarrow(a \rightarrow(a \rightarrow a)) \stackrel{2.7(1)}{=} a \rightarrow(b \rightarrow(a \rightarrow a)) \stackrel{2.7(1)}{=}$ $a \rightarrow(a \rightarrow(b \rightarrow a))$. Observe that $((a \rightarrow a) \rightarrow b) \rightarrow a=t_{1}(a, a, b, a)$ $\stackrel{(5.4)}{=} t_{2}(a, a, b, a)=b \rightarrow((a \rightarrow a) \rightarrow a) \stackrel{2.11}{=} b \rightarrow(a \rightarrow(a \rightarrow a)) \stackrel{2.7(1)}{=} a \rightarrow$ $(b \rightarrow(a \rightarrow a)) \stackrel{2.7(1)}{=} a \rightarrow(a \rightarrow(b \rightarrow a))$. Consequently, $\mathbf{A} \models(42 B 15)$. From $(a \rightarrow a) \rightarrow(b \rightarrow a)=t_{5}(b, a, a, a)=t_{2}(b, a, a, a)=a \rightarrow((a \rightarrow b) \rightarrow a)$, we have that $\mathbf{A} \models(42 B 23)$. Since $a \rightarrow((a \rightarrow b) \rightarrow a)=t_{2}(b, a, a, a)=$ $t_{4}(b, a, a, a)=((a \rightarrow a) \rightarrow b) \rightarrow a$ we conclude that $\mathbf{A} \models(42 B 25)$.

Since $\mathbf{A} \models 0 \rightarrow x \approx x$, by Lemma 2.6,

$$
\mathbf{A} \models(x \rightarrow y)^{\prime} \approx x^{\prime} \rightarrow y^{\prime} .
$$

Since $(a \rightarrow(b \rightarrow a)) \rightarrow a \stackrel{2.7(2)}{=} a^{\prime} \rightarrow(a \rightarrow(b \rightarrow a))^{\prime} \stackrel{2.7(1)}{=} a^{\prime} \rightarrow(b \rightarrow(a \rightarrow a))^{\prime}$ $\stackrel{(5.5)}{=} a^{\prime} \rightarrow\left(b^{\prime} \rightarrow(a \rightarrow a)^{\prime}\right) \stackrel{(5.5)}{=} a^{\prime} \rightarrow\left(b^{\prime} \rightarrow\left(a^{\prime} \rightarrow a^{\prime}\right)\right) \stackrel{2.7(2)}{=} a^{\prime} \rightarrow\left(b^{\prime} \rightarrow(a \rightarrow\right.$ $a) \stackrel{2.7(1)}{=} b^{\prime} \rightarrow\left(a^{\prime} \rightarrow(a \rightarrow a)\right) \stackrel{2.4(11)}{=} b^{\prime} \rightarrow(a \rightarrow a) \stackrel{2.4(7)}{=} b^{\prime} \rightarrow(a \rightarrow(a \rightarrow a))$ $\stackrel{2.7(1)}{=} a \rightarrow\left(b^{\prime} \rightarrow(a \rightarrow a)\right) \stackrel{2.7(2)}{=} a \rightarrow\left(b^{\prime} \rightarrow\left(a^{\prime} \rightarrow a^{\prime}\right)\right) \stackrel{2.7(1)}{=} a \rightarrow\left(a^{\prime} \rightarrow\left(b^{\prime} \rightarrow\right.\right.$ $\left.\left.a^{\prime}\right)\right) \stackrel{(5.5)}{=} a \rightarrow\left(a^{\prime} \rightarrow(b \rightarrow a)^{\prime}\right) \stackrel{2.7(2)}{=} a \rightarrow((b \rightarrow a) \rightarrow a)$. A satisfies the identity (42D24). Notice that A satisfies the identity (42D45), in view of $((a \rightarrow b) \rightarrow a) \rightarrow a=t_{1}(a, b, a, a) \stackrel{(5.4)}{=} t_{2}(a, b, a, a)=a \rightarrow((b \rightarrow a) \rightarrow a)$ $\stackrel{\text { using }}{=} \stackrel{(42 D 24)}{=}(a \rightarrow(b \rightarrow a)) \rightarrow a$.

Since $((a \rightarrow b) \rightarrow b) \rightarrow a=t_{4}(b, b, a, a)=t_{2}(b, b, a, a)=a \rightarrow((b \rightarrow b) \rightarrow$ $a)$, the algebra $\mathbf{A} \models(42 F 25)$. Also, since $((a \rightarrow b) \rightarrow b) \rightarrow b=t_{4}(b, b, a, b)$ $\stackrel{(5.4)}{=} t_{2}(b, b, a, b)=a \rightarrow((b \rightarrow b) \rightarrow b) \stackrel{2.11}{=} a \rightarrow(b \rightarrow(b \rightarrow b))$, we have that $\mathbf{A} \models(42 G 15)$.

Recall that the varieties $42 \mathcal{A} 24,42 \mathcal{A} 45,42 \mathcal{B} 13,42 \mathcal{B} 15,42 \mathcal{B} 23,42 \mathcal{B} 25$, $42 \mathcal{D} 24,42 \mathcal{D} 45,42 \mathcal{F} 25$, and $42 \mathcal{G} 15$ are defined, respectively, by the following:

$(42 \mathrm{~A} 24) x \rightarrow((x \rightarrow x) \rightarrow y) \approx(x \rightarrow(x \rightarrow x)) \rightarrow y$
$(42 \mathrm{~A} 45)(x \rightarrow(x \rightarrow x)) \rightarrow y \approx((x \rightarrow x) \rightarrow x) \rightarrow y$
$(42 \mathrm{~B} 13) x \rightarrow(x \rightarrow(y \rightarrow x)) \approx(x \rightarrow x) \rightarrow(y \rightarrow x)$
$(42 \mathrm{~B} 15) x \rightarrow(x \rightarrow(y \rightarrow x)) \approx((x \rightarrow x) \rightarrow y) \rightarrow x$
$(42 \mathrm{~B} 23) x \rightarrow((x \rightarrow y) \rightarrow x) \approx(x \rightarrow x) \rightarrow(y \rightarrow x)$
$(42 \mathrm{~B} 25) x \rightarrow((x \rightarrow y) \rightarrow x) \approx((x \rightarrow x) \rightarrow y) \rightarrow x$
$(42 \mathrm{D} 24) x \rightarrow((y \rightarrow x) \rightarrow x) \approx(x \rightarrow(y \rightarrow x)) \rightarrow x$
$(42 \mathrm{D} 45)(x \rightarrow(y \rightarrow x)) \rightarrow x \approx((x \rightarrow y) \rightarrow x) \rightarrow x$
$(42 \mathrm{~F} 25) x \rightarrow((y \rightarrow y) \rightarrow x) \approx((x \rightarrow y) \rightarrow y) \rightarrow x$ 
$(42 \mathrm{G} 15) x \rightarrow(y \rightarrow(y \rightarrow y)) \approx((x \rightarrow y) \rightarrow y) \rightarrow y$

Using Lemma 5.5 and Theorem 5.6, one can easily verify the following Theorem.

THEOREM 5.7. $42 \mathcal{A} 24=42 \mathcal{A} 45=42 \mathcal{B} 13=42 \mathcal{B} 15=42 \mathcal{B} 23=42 \mathcal{B} 25=$ $42 \mathcal{D} 24=42 \mathcal{D} 45=42 \mathcal{F} 25=42 \mathcal{G} 15$.

LEMMA 5.8. $42 \mathcal{A} 24=31 \mathcal{A} 12$.

Proof. Let $\mathbf{A} \in 31 \mathcal{A} 12$. Since, replacing $x:=0$ in the identity (31A12), we obtain $0=0^{\prime}$ then $a=a^{\prime \prime}=a^{\prime} \rightarrow 0=a^{\prime} \rightarrow 0^{\prime}=0 \rightarrow a$ by Lemma 2.3 (2). Then, by Lemma 2.13, $\mathbf{A} \in 42 \mathcal{A} 24$. Therefore $31 \mathcal{A} 12 \subseteq 42 \mathcal{A} 24$.

For the converse let consider $\mathbf{A} \in 42 \mathcal{A} 24$. Let $a \in A$. By Lemma 5.5

$$
\mathbf{A} \models 0 \rightarrow x \approx x
$$

Hence, $(a \rightarrow a) \rightarrow a \stackrel{2.4(4)}{=}(0 \rightarrow a) \rightarrow a \stackrel{(5.6)}{=} a \rightarrow a \stackrel{2.4(7)}{=} a \rightarrow(a \rightarrow a)$.

Let $J$ be the set consisting of the following identities: (42B12), (42B35), (42C23), (42C25), (42C35) and (42G25).

LEMMA 5.9. Let $(x) \in J$. If $\mathbf{A} \models(x)$ then $A \models 0 \rightarrow(x \rightarrow x) \approx x \rightarrow x$.

Proof. Let $a \in A$. Consider the following cases:

Case 1: $\mathbf{A}=(42 B 12)$. Then $a \rightarrow a \stackrel{2.2(4)}{=} a \rightarrow\left(a^{\prime} \rightarrow a\right)=a \rightarrow((a \rightarrow 0) \rightarrow a)$ $\stackrel{(42 B 12)}{=} a \rightarrow(a \rightarrow(0 \rightarrow a)) \stackrel{2.7(1)}{=} a \rightarrow(0 \rightarrow(a \rightarrow a)) \stackrel{2.7(1)}{=} 0 \rightarrow(a \rightarrow(a \rightarrow a))$ $\stackrel{2.4(7)}{=} 0 \rightarrow(a \rightarrow a)$.

Case 2: $(x)=(42 B 35)$. Hence $0 \rightarrow(a \rightarrow a) \stackrel{2.4(6) \operatorname{and}(9)}{=}(0 \rightarrow a) \rightarrow(0 \rightarrow a)$ $\stackrel{2.7(1)}{=} 0 \rightarrow((0 \rightarrow a) \rightarrow a) \stackrel{2.4(4)}{=} 0 \rightarrow((a \rightarrow a) \rightarrow a) \stackrel{2.7(1)}{=}(a \rightarrow a) \rightarrow(0 \rightarrow a)$ $\stackrel{(42 B 35)}{=}((a \rightarrow a) \rightarrow 0) \rightarrow a=(a \rightarrow a)^{\prime} \rightarrow a \stackrel{2.7(2)}{=} a^{\prime} \rightarrow(a \rightarrow a) \stackrel{2.4(11)}{=} a \rightarrow a$.

Case 3: $(x)=(42 C 23)$. We get $a \rightarrow a \stackrel{2.2(1)}{=} 0^{\prime} \rightarrow(a \rightarrow a)=(0 \rightarrow$ $0) \rightarrow(a \rightarrow a) \stackrel{(42 C 23)}{=} 0 \rightarrow((0 \rightarrow a) \rightarrow a) \stackrel{2.7(1)}{=}(0 \rightarrow a) \rightarrow(0 \rightarrow a)$ $\stackrel{2.4(6) \operatorname{and}(9)}{=} 0 \rightarrow(a \rightarrow a)$.

Case 4: $(x)=(42 C 25)$. One has $a \rightarrow a \stackrel{2.2(1)}{=}\left(0^{\prime} \rightarrow a\right) \rightarrow a=((0 \rightarrow$ $0) \rightarrow a) \rightarrow a \stackrel{(42 C 25)}{=} 0 \rightarrow((0 \rightarrow a) \rightarrow a) \stackrel{2.7(1)}{=}(0 \rightarrow a) \rightarrow(0 \rightarrow a)$ $\stackrel{2.4(6) \operatorname{and}(9)}{=} 0 \rightarrow(a \rightarrow a)$. 
Case 5: $(x)=(42 C 35)$. Then $0 \rightarrow(a \rightarrow a)^{\prime} \stackrel{2.7(2)}{=}(a \rightarrow a) \rightarrow 0^{\prime}=(a \rightarrow a) \rightarrow$ $(0 \rightarrow 0) \stackrel{(42 C 35)}{=}((a \rightarrow a) \rightarrow 0) \rightarrow 0 \stackrel{x \approx x^{\prime \prime}}{=} a \rightarrow a$. Therefore,

$$
\mathbf{A} \models 0 \rightarrow(x \rightarrow x)^{\prime} \approx x \rightarrow x .
$$

Hence $a \rightarrow a \stackrel{(5.7)}{=} 0 \rightarrow(a \rightarrow a)^{\prime} \stackrel{(5.7)}{=} 0 \rightarrow\left(0 \rightarrow(a \rightarrow a)^{\prime}\right)^{\prime} \stackrel{2.4(8)}{=} 0 \rightarrow(a \rightarrow a)^{\prime \prime}$ $\stackrel{x \approx x^{\prime \prime}}{=} 0 \rightarrow(a \rightarrow a)$.

Case 6: $(x)=(42 G 25)$. We have $0 \rightarrow(a \rightarrow a) \stackrel{2.4(6) \operatorname{and}(9)}{=}(0 \rightarrow a) \rightarrow(0 \rightarrow a)$ $\stackrel{2.7(1)}{=} 0 \rightarrow((0 \rightarrow a) \rightarrow a) \stackrel{2.4(4)}{=} 0 \rightarrow((a \rightarrow a) \rightarrow a) \stackrel{(42 G 25)}{=}((0 \rightarrow a) \rightarrow a) \rightarrow a$ $\stackrel{2.4(2)}{=} a \rightarrow a$, which proves the lemma.

THEOREM 5.10. If $\mathbf{A} \models 0 \rightarrow(x \rightarrow x) \approx x \rightarrow x$ then $\mathbf{A} \models(y)$, for all $(y) \in J$.

Proof. Let $a, b \in A$. By Lemma 2.9 (1) and (2),

$$
\mathbf{A} \models(x \rightarrow x) \rightarrow y^{\prime} \approx((x \rightarrow x) \rightarrow y)^{\prime}
$$

and

$$
\mathbf{A} \models(x \rightarrow x) \rightarrow(y \rightarrow z) \approx((x \rightarrow x) \rightarrow y) \rightarrow z
$$

Using (5.9) we have that A satisfies (42B35) and (42C35).

Since $a \rightarrow((a \rightarrow b) \rightarrow a) \stackrel{2.7(1)}{=}(a \rightarrow b) \rightarrow(a \rightarrow a) \stackrel{(I)}{=}\left(\left((a \rightarrow a)^{\prime} \rightarrow a\right) \rightarrow\right.$ $\left.(b \rightarrow(a \rightarrow a))^{\prime}\right)^{\prime}=\left((((a \rightarrow a) \rightarrow 0) \rightarrow a) \rightarrow(b \rightarrow(a \rightarrow a))^{\prime}\right)^{\prime} \stackrel{(5.9)}{=}(((a \rightarrow$ $\left.a) \rightarrow(0 \rightarrow a)) \rightarrow(b \rightarrow(a \rightarrow a))^{\prime}\right)^{\prime} \stackrel{2.7(1)}{=}((0 \rightarrow((a \rightarrow a) \rightarrow a)) \rightarrow(b \rightarrow$ $\left.(a \rightarrow a))^{\prime}\right)^{\prime} \stackrel{2.4(6) \operatorname{and}(9)}{=}\left(((0 \rightarrow(a \rightarrow a)) \rightarrow(0 \rightarrow a)) \rightarrow(b \rightarrow(a \rightarrow a))^{\prime}\right)^{\prime}$ $\stackrel{\text { hyp }}{=}\left(((a \rightarrow a) \rightarrow(0 \rightarrow a)) \rightarrow(b \rightarrow(a \rightarrow a))^{\prime}\right)^{\prime} \stackrel{2.7(1)}{=}((0 \rightarrow((a \rightarrow a) \rightarrow$ $\left.a)) \rightarrow(b \rightarrow(a \rightarrow a))^{\prime}\right)^{\prime} \stackrel{2.4(4)}{=}\left((0 \rightarrow((0 \rightarrow a) \rightarrow a)) \rightarrow(b \rightarrow(a \rightarrow a))^{\prime}\right)^{\prime}$ $\stackrel{2.7(1)}{=}\left(((0 \rightarrow a) \rightarrow(0 \rightarrow a)) \rightarrow(b \rightarrow(a \rightarrow a))^{\prime}\right)^{\prime} \stackrel{(5.8)}{=}(((0 \rightarrow a) \rightarrow(0 \rightarrow$ $a)) \rightarrow(b \rightarrow(a \rightarrow a)))^{\prime \prime} \stackrel{x \approx x^{\prime \prime}}{=}((0 \rightarrow a) \rightarrow(0 \rightarrow a)) \rightarrow(b \rightarrow(a \rightarrow a))$ $\stackrel{2.4(6) \operatorname{and}(9)}{=}(0 \rightarrow(a \rightarrow a)) \rightarrow(b \rightarrow(a \rightarrow a)) \stackrel{\text { hyp }}{=}(a \rightarrow a) \rightarrow(b \rightarrow(a \rightarrow a))$ $\stackrel{2.7(1)}{=} b \rightarrow((a \rightarrow a) \rightarrow(a \rightarrow a)) \stackrel{2.4(12)}{=} b \rightarrow(a \rightarrow a) \stackrel{2.4(7)}{=} b \rightarrow(a \rightarrow(a \rightarrow a))$ $\stackrel{2.7(1)}{=} a \rightarrow(b \rightarrow(a \rightarrow a)) \stackrel{2.7(1)}{=} a \rightarrow(a \rightarrow(b \rightarrow a))$, the algebra A satisfies (42B12). By Lemma 2.9 (3) and Lemma 2.7 (1), A satisfies (42C23). In view 
of $(5.9)$ and $(42 \mathrm{C} 23)$, we have $\mathbf{A} \models(x \rightarrow x) \rightarrow(y \rightarrow y) \approx x \rightarrow((x \rightarrow y) \rightarrow$ $y)$ proving that the identity $(42 \mathrm{C} 25)$ holds in $\mathbf{A}$.

It remains to verify that the algebra A satisfies identity (42G25). To finish off the proof, $((a \rightarrow b) \rightarrow b) \rightarrow b \stackrel{(I)}{=}\left(\left(b^{\prime} \rightarrow a\right) \rightarrow(b \rightarrow b)^{\prime}\right)^{\prime} \rightarrow b$ $\stackrel{2.7(2)}{=} b^{\prime} \rightarrow\left(\left(b^{\prime} \rightarrow a\right) \rightarrow(b \rightarrow b)^{\prime}\right)^{\prime \prime} \stackrel{x^{\prime \prime}}{=}{ }^{x} b^{\prime} \rightarrow\left(\left(b^{\prime} \rightarrow a\right) \rightarrow(b \rightarrow b)^{\prime}\right)$ $\stackrel{2.7(2)}{=} b^{\prime} \rightarrow\left((b \rightarrow b) \rightarrow\left(b^{\prime} \rightarrow a\right)^{\prime}\right) \stackrel{2.7(1)}{=}(b \rightarrow b) \rightarrow\left(b^{\prime} \rightarrow\left(b^{\prime} \rightarrow a\right)^{\prime}\right) \stackrel{2.7(2)}{=}(b \rightarrow$ $b) \rightarrow\left(\left(b^{\prime} \rightarrow a\right) \rightarrow b\right) \stackrel{(I)}{=}(b \rightarrow b) \rightarrow\left(\left(b^{\prime} \rightarrow b^{\prime}\right) \rightarrow(a \rightarrow b)^{\prime}\right)^{\prime} \stackrel{2.7(2)}{=}(b \rightarrow b) \rightarrow$ $\left((b \rightarrow b) \rightarrow(a \rightarrow b)^{\prime}\right)^{\prime} \stackrel{(5.8)}{=}(b \rightarrow b) \rightarrow((b \rightarrow b) \rightarrow(a \rightarrow b))^{\prime \prime} \stackrel{x^{\prime \prime} \approx x}{=}(b \rightarrow b) \rightarrow$ $((b \rightarrow b) \rightarrow(a \rightarrow b)) \stackrel{2.4(7)}{=}(b \rightarrow b) \rightarrow(a \rightarrow b) \stackrel{2.7(1)}{=} a \rightarrow((b \rightarrow b) \rightarrow b)$. Consequently, $\mathbf{A} \models(42 G 25)$.

Recall that the varieties $42 \mathcal{B} 12,42 \mathcal{B} 35,42 \mathcal{C} 23,42 \mathcal{C} 25,42 \mathcal{C} 35$, and $42 \mathcal{G} 25$ are defined, respectively, by the following:

(42B12) $x \rightarrow(x \rightarrow(y \rightarrow x)) \approx x \rightarrow((x \rightarrow y) \rightarrow x)$ (42B35) $(x \rightarrow x) \rightarrow(y \rightarrow x) \approx((x \rightarrow x) \rightarrow y) \rightarrow x$ $(42 \mathrm{C} 23) x \rightarrow((x \rightarrow y) \rightarrow y) \approx(x \rightarrow x) \rightarrow(y \rightarrow y)$ $(42 \mathrm{C} 25) x \rightarrow((x \rightarrow y) \rightarrow y) \approx((x \rightarrow x) \rightarrow y) \rightarrow y$ $(42 \mathrm{C} 35)(x \rightarrow x) \rightarrow(y \rightarrow y) \approx((x \rightarrow x) \rightarrow y) \rightarrow y$ $(42 \mathrm{G} 25) x \rightarrow((y \rightarrow y) \rightarrow y) \approx((x \rightarrow y) \rightarrow y) \rightarrow y$

In view of Lemma 5.9 and Theorem 5.10, the following theorem is immediate.

THEOREM 5.11. $42 \mathcal{B} 12=42 \mathcal{B} 35=42 \mathcal{C} 23=42 \mathcal{C} 25=42 \mathcal{C} 35=42 \mathcal{G} 25$.

LEMMA 5.12. $42 \mathcal{A} 12=42 \mathcal{D} 23=42 \mathcal{D} 35$.

Proof. In view of Lemma 2.14 (7), we have

$$
\mathbf{A} \models x \rightarrow((y \rightarrow x) \rightarrow x) \approx((x \rightarrow y) \rightarrow x) \rightarrow x .
$$

Hence,

$$
\mathbf{A} \models(42 D 23) \text { if and only if } \mathbf{A} \models(42 D 35),
$$

proving that $42 \mathcal{D} 23=42 \mathcal{D} 35$.

Assume $\mathbf{A} \in 42 \mathcal{A} 12$ and $a, b \in A$. Observe that $((a \rightarrow b) \rightarrow a) \rightarrow a \stackrel{2.4(3)}{=}$ $\left(a \rightarrow(b \rightarrow a)^{\prime}\right)^{\prime} \rightarrow a \stackrel{2.7(2)}{=} a^{\prime} \rightarrow\left(a \rightarrow(b \rightarrow a)^{\prime}\right) \stackrel{2.7(2)}{=} a^{\prime} \rightarrow\left((b \rightarrow a) \rightarrow a^{\prime}\right)$ 


$$
\begin{aligned}
& \stackrel{2.7(1)}{=}(b \rightarrow a) \rightarrow\left(a^{\prime} \rightarrow a^{\prime}\right) \stackrel{2.7(2)}{=}(b \rightarrow a) \rightarrow(a \rightarrow a) \stackrel{2.7(2)}{=}\left(a^{\prime} \rightarrow b^{\prime}\right) \rightarrow(a \rightarrow a) \\
& 2.4(7) \\
& \stackrel{2}{=}\left(a^{\prime} \rightarrow\left(a^{\prime} \rightarrow b^{\prime}\right)\right) \rightarrow(a \rightarrow a) \stackrel{2.4(7)}{=}\left(a^{\prime} \rightarrow\left(a^{\prime} \rightarrow\left(a^{\prime} \rightarrow b^{\prime}\right)\right) \rightarrow(a \rightarrow a)\right. \\
& \stackrel{(42 A 12)}{=}\left(a^{\prime} \rightarrow\left(\left(a^{\prime} \rightarrow a^{\prime}\right) \rightarrow b\right)\right) \rightarrow(a \rightarrow a) \stackrel{2.7(1)}{=}\left(\left(a^{\prime} \rightarrow a^{\prime}\right) \rightarrow\left(a^{\prime} \rightarrow b\right)\right) \rightarrow \\
& (a \rightarrow a) \stackrel{2.7(2)}{=}\left((a \rightarrow a) \rightarrow\left(a^{\prime} \rightarrow b\right)\right) \rightarrow(a \rightarrow a) \stackrel{2.4(4)}{=}\left(0 \rightarrow\left(a^{\prime} \rightarrow b\right)\right) \rightarrow \\
& (a \rightarrow a) \stackrel{2.7(1)}{=}\left(a^{\prime} \rightarrow(0 \rightarrow b)\right) \rightarrow(a \rightarrow a) \stackrel{2.7(2)}{=}\left((0 \rightarrow b)^{\prime} \rightarrow a\right) \rightarrow(a \rightarrow a) \\
& \stackrel{2.4(13)}{=}\left(0 \rightarrow(0 \rightarrow b)^{\prime \prime}\right) \rightarrow(a \rightarrow a) \stackrel{x \approx x^{\prime \prime}}{=}(0 \rightarrow(0 \rightarrow b)) \rightarrow(a \rightarrow a) \stackrel{2.4(7)}{=} \\
& (0 \rightarrow b) \rightarrow(a \rightarrow a) \stackrel{2.7(2)}{=}(0 \rightarrow b) \rightarrow\left(a^{\prime} \rightarrow a^{\prime}\right) \stackrel{x \approx x^{\prime \prime}}{=}\left(0 \rightarrow b^{\prime \prime}\right) \rightarrow\left(a^{\prime} \rightarrow a^{\prime}\right) \\
& \stackrel{2.4(13)}{=}\left(b^{\prime} \rightarrow a^{\prime}\right) \rightarrow\left(a^{\prime} \rightarrow a^{\prime}\right) \stackrel{2.7(2)}{=}(a \rightarrow b) \rightarrow(a \rightarrow a) . \text { Hence } \mathbf{A} \in 42 \mathcal{D} 35 .
\end{aligned}
$$

Now assume that $\mathbf{A} \in 42 \mathcal{D} 35$ and $a, b \in A$. Since $0 \rightarrow a \stackrel{x \approx x^{\prime \prime}}{=}(0 \rightarrow a)^{\prime \prime}$ $=((0 \rightarrow a) \rightarrow 0) \rightarrow 0 \stackrel{(42 D 35)}{=}(0 \rightarrow a) \rightarrow(0 \rightarrow 0)=(0 \rightarrow a) \rightarrow 0^{\prime}$ $\stackrel{2.7(2)}{=} 0 \rightarrow(0 \rightarrow a)^{\prime} \stackrel{2.4(8)}{=} 0 \rightarrow a^{\prime}$, the algebra $\mathbf{A}$ satisfies

$$
0 \rightarrow x \approx 0 \rightarrow x^{\prime}
$$

Therefore, $a \rightarrow((a \rightarrow a) \rightarrow b) \stackrel{2.7(1)}{=}(a \rightarrow a) \rightarrow(a \rightarrow b) \stackrel{2.4(13)}{=}\left(0 \rightarrow a^{\prime}\right) \rightarrow$ $(a \rightarrow b) \stackrel{2.7(1)}{=} a \rightarrow\left(\left(0 \rightarrow a^{\prime}\right) \rightarrow b\right) \stackrel{(5.10)}{=} a \rightarrow((0 \rightarrow a) \rightarrow b) \stackrel{2.4(4)}{=} a \rightarrow$ $((b \rightarrow a) \rightarrow b) \stackrel{2.7(1)}{=}(b \rightarrow a) \rightarrow(a \rightarrow b) \stackrel{2.4(12)}{=} a \rightarrow b \stackrel{2.4(7)}{=} a \rightarrow(a \rightarrow b)$ $\stackrel{2.4(7)}{=} a \rightarrow(a \rightarrow(a \rightarrow b))$. Hence $\mathbf{A} \in 42 \mathcal{A} 12$.

Let $K$ be the set consisting of the following identities: (42C12), (42C13), (42C15), (42D12) and (42F35).

LEMMA 5.13. Let $(x) \in K$. If $\mathbf{A} \models(x)$ then $\mathbf{A} \models 0 \rightarrow x \approx x \rightarrow x$.

Proof. Let $a \in A$.

If $\mathbf{A}=(42 C 12), a \rightarrow a \stackrel{2.7(2)}{=} a^{\prime} \rightarrow a^{\prime} \stackrel{x^{\prime \prime} \approx x}{=} a^{\prime} \rightarrow a^{\prime \prime \prime}=a^{\prime} \rightarrow\left(\left(a^{\prime} \rightarrow 0\right) \rightarrow 0\right)$ $\stackrel{(42 C 12)}{=} a^{\prime} \rightarrow\left(a^{\prime} \rightarrow(0 \rightarrow 0)\right)=a^{\prime} \rightarrow\left(a^{\prime} \rightarrow 0^{\prime}\right) \stackrel{2.4(7)}{=} a^{\prime} \rightarrow 0^{\prime} \stackrel{2.3(1)}{=} 0 \rightarrow a$.

If $\mathbf{A} \models(42 C 13), 0 \rightarrow a \stackrel{2.7(2)}{=} a^{\prime} \rightarrow 0^{\prime} \stackrel{2.4(7)}{=} a^{\prime} \rightarrow\left(a^{\prime} \rightarrow 0^{\prime}\right)=a^{\prime} \rightarrow\left(a^{\prime} \rightarrow\right.$ $(0 \rightarrow 0)) \stackrel{(42 C 13)}{=}\left(a^{\prime} \rightarrow a^{\prime}\right) \rightarrow(0 \rightarrow 0)=\left(a^{\prime} \rightarrow a^{\prime}\right) \rightarrow 0^{\prime} \stackrel{2.3(2)}{=} 0 \rightarrow\left(a^{\prime} \rightarrow a^{\prime}\right)^{\prime}$ $\stackrel{2.4(10)}{=} 0 \rightarrow\left(a^{\prime \prime} \rightarrow a^{\prime \prime}\right) \stackrel{x^{\prime \prime}}{=} 0 x \rightarrow(a \rightarrow a) \stackrel{2.4(7)}{=} 0 \rightarrow(0 \rightarrow(a \rightarrow a))$ $\stackrel{(42 C 13)}{=}(0 \rightarrow 0) \rightarrow(a \rightarrow a)=0^{\prime} \rightarrow(a \rightarrow a) \stackrel{2.2(1)}{=} a \rightarrow a$. 
If $\mathbf{A} \models(42 C 15), 0 \rightarrow a \stackrel{2.7(2)}{=} a^{\prime} \rightarrow 0^{\prime} \stackrel{2.4(7)}{=} a^{\prime} \rightarrow\left(a^{\prime} \rightarrow 0^{\prime}\right)=a^{\prime} \rightarrow\left(a^{\prime} \rightarrow\right.$ $(0 \rightarrow 0)) \stackrel{(42 C 15)}{=}\left(\left(a^{\prime} \rightarrow a^{\prime}\right) \rightarrow 0\right) \rightarrow 0 \stackrel{x^{\prime \prime} \approx x}{=} a^{\prime} \rightarrow a^{\prime} \stackrel{2.7(2)}{=} a \rightarrow a$.

If $\mathbf{A} \models(42 D 12), 0 \rightarrow a \stackrel{2.7(2)}{=} a^{\prime} \rightarrow 0^{\prime} \stackrel{2.2(4)}{=} a^{\prime} \rightarrow\left(0 \rightarrow 0^{\prime}\right)=a^{\prime} \rightarrow$ $(0 \rightarrow(0 \rightarrow 0)) \stackrel{2.7(1)}{=} 0 \rightarrow\left(a^{\prime} \rightarrow(0 \rightarrow 0)\right) \stackrel{(42 D 12)}{=} 0 \rightarrow\left(\left(a^{\prime} \rightarrow 0\right) \rightarrow 0\right)$ $\stackrel{x^{\prime \prime} \cong x}{=} 0 \rightarrow(a \rightarrow 0)=0 \rightarrow a^{\prime}$.

Hence,

$$
\mathbf{A} \models 0 \rightarrow x \approx 0 \rightarrow x^{\prime}
$$

Therefore, $a \rightarrow a \stackrel{2.2(4)}{=} a \rightarrow\left(a^{\prime} \rightarrow a\right) \stackrel{2.2(4)}{=} a \rightarrow\left(\left(a \rightarrow a^{\prime}\right) \rightarrow a\right) \stackrel{2.4(4)}{=} a \rightarrow$ $\left(\left(0 \rightarrow a^{\prime}\right) \rightarrow a\right) \stackrel{(5.11)}{=} a \rightarrow((0 \rightarrow a) \rightarrow a) \stackrel{(42 D 12)}{=} a \rightarrow(0 \rightarrow(a \rightarrow a))$

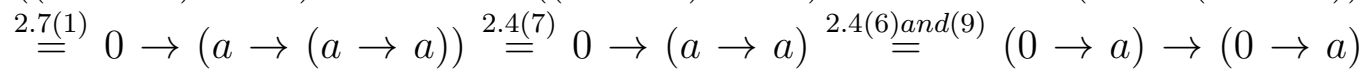
$\stackrel{(5.11)}{=}\left(0 \rightarrow a^{\prime}\right) \rightarrow(0 \rightarrow a) \stackrel{2.4(6) \operatorname{and}(9)}{=} 0 \rightarrow\left(a^{\prime} \rightarrow a\right) \stackrel{2.2(4)}{=} 0 \rightarrow a$.

If $\mathbf{A} \models(42 F 35), 0 \rightarrow a \stackrel{2.4(11)}{=} a^{\prime} \rightarrow(0 \rightarrow a)=(a \rightarrow 0) \rightarrow(0 \rightarrow a)$ $\stackrel{(42 F 35)}{=}[(a \rightarrow 0) \rightarrow 0] \rightarrow a=a^{\prime \prime} \rightarrow a \stackrel{x^{\prime \prime}}{=} x . a \rightarrow a$.

THEOREM 5.14. If $\mathbf{A} \models 0 \rightarrow x \approx x \rightarrow x$ then $\mathbf{A} \models(y)$ for all $(y) \in K$.

Proof. Let $a, b \in A$. By the hypothesis and Lemma 2.8 (1) A satisfies

$$
0 \rightarrow(x \rightarrow x) \approx x \rightarrow x .
$$

Hence, by Lemma 2.9 (3) and Lemma 2.7 (1), the algebra A satisfies (42C23).

In view of Theorem 5.11, the algebra A satisfies the identity (42B12) too.

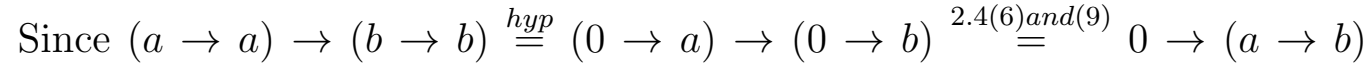
$\stackrel{2.7(1)}{=} a \rightarrow(0 \rightarrow b) \stackrel{\text { hyp }}{=} a \rightarrow(b \rightarrow b) \stackrel{2.4(7)}{=} a \rightarrow(a \rightarrow(b \rightarrow b))$, the algebra A satisfies (42C13). Therefore, $a \rightarrow((a \rightarrow b) \rightarrow b) \stackrel{(42 C 23)}{=}(a \rightarrow a) \rightarrow(b \rightarrow b)$ $\stackrel{(42 C 13)}{=} a \rightarrow(a \rightarrow(b \rightarrow b))$. Consequently, $\mathbf{A} \models(42 C 12)$. By Lemma $2.9(2)$,

$$
\mathbf{A} \models(x \rightarrow x) \rightarrow(y \rightarrow z) \approx((x \rightarrow x) \rightarrow y) \rightarrow z
$$

Therefore, $a \rightarrow(a \rightarrow(b \rightarrow b)) \stackrel{(42 C 12)}{=} a \rightarrow((a \rightarrow b) \rightarrow b) \stackrel{2.7(1)}{=}(a \rightarrow b) \rightarrow$

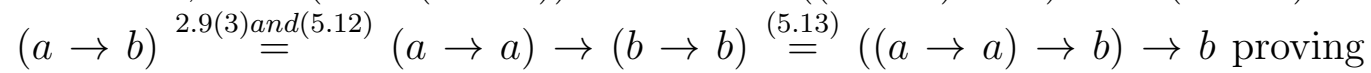
(42C15). By Lemma 2.8 (2) A satisfies

$$
0 \rightarrow x^{\prime} \approx 0 \rightarrow x
$$


Since $a \rightarrow((b \rightarrow a) \rightarrow a) \stackrel{2.7(1)}{=}(b \rightarrow a) \rightarrow(a \rightarrow a) \stackrel{h y p}{=}(b \rightarrow a) \rightarrow(0 \rightarrow a)$ $\stackrel{(I)}{=}\left(\left((0 \rightarrow a)^{\prime} \rightarrow b\right) \rightarrow(a \rightarrow(0 \rightarrow a))^{\prime}\right)^{\prime} \stackrel{2.7(1)}{=}\left(\left((0 \rightarrow a)^{\prime} \rightarrow b\right) \rightarrow(0 \rightarrow(a \rightarrow\right.$ $\left.a))^{\prime}\right)^{\prime} \stackrel{(5.12)}{=}\left(\left((0 \rightarrow a)^{\prime} \rightarrow b\right) \rightarrow(a \rightarrow a)^{\prime}\right)^{\prime} \stackrel{\text { hyp }}{=}\left(\left((0 \rightarrow a)^{\prime} \rightarrow b\right) \rightarrow(0 \rightarrow a)^{\prime}\right)^{\prime}$ $\stackrel{2.2(1)}{=}\left(\left((0 \rightarrow a)^{\prime} \rightarrow b\right) \rightarrow\left(0^{\prime} \rightarrow(0 \rightarrow a)\right)^{\prime}\right)^{\prime} \stackrel{(I)}{=}\left(b \rightarrow 0^{\prime}\right) \rightarrow(0 \rightarrow a)$ $\stackrel{2.3(2)}{=}\left(0 \rightarrow b^{\prime}\right) \rightarrow(0 \rightarrow a) \stackrel{(5.14)}{=}(0 \rightarrow b) \rightarrow(0 \rightarrow a) \stackrel{\text { hyp }}{=}(0 \rightarrow b) \rightarrow(a \rightarrow a)$ $\stackrel{2.7(1)}{=} a \rightarrow((0 \rightarrow b) \rightarrow a) \stackrel{2.4(4)}{=} a \rightarrow((a \rightarrow b) \rightarrow a) \stackrel{(42 B 12)}{=} a \rightarrow(a \rightarrow(b \rightarrow$ a) $\stackrel{2.7(1)}{=} a \rightarrow(b \rightarrow(a \rightarrow a))$, the identity (42D12) holds in A. Finally, $\mathbf{A}=(42 F 35)$, in view of the following derivation: $((a \rightarrow b) \rightarrow b) \rightarrow a$ $\stackrel{(I)}{=}\left(\left(a^{\prime} \rightarrow(a \rightarrow b)\right) \rightarrow(b \rightarrow a)^{\prime}\right)^{\prime} \stackrel{2.7(1)}{=}\left(\left(a \rightarrow\left(a^{\prime} \rightarrow b\right)\right) \rightarrow(b \rightarrow a)^{\prime}\right)^{\prime}$ $\stackrel{2.7(2)}{=}\left(\left(a \rightarrow\left(b^{\prime} \rightarrow a\right)\right) \rightarrow(b \rightarrow a)^{\prime}\right)^{\prime} \stackrel{2.7(1)}{=}\left(\left(b^{\prime} \rightarrow(a \rightarrow a)\right) \rightarrow(b \rightarrow a)^{\prime}\right)^{\prime}$ $\stackrel{\text { hyp }}{=}\left(\left(b^{\prime} \rightarrow(0 \rightarrow a)\right) \rightarrow(b \rightarrow a)^{\prime}\right)^{\prime} \stackrel{2.7(1)}{=}\left(\left(0 \rightarrow\left(b^{\prime} \rightarrow a\right)\right) \rightarrow(b \rightarrow a)^{\prime}\right)^{\prime}$ $\stackrel{2.4(6) \operatorname{and}(9)}{=}\left(\left(\left(0 \rightarrow b^{\prime}\right) \rightarrow(0 \rightarrow a)\right) \rightarrow(b \rightarrow a)^{\prime}\right)^{\prime} \stackrel{(5.14)}{=}(((0 \rightarrow b) \rightarrow(0 \rightarrow a)) \rightarrow$ $\left.(b \rightarrow a)^{\prime}\right)^{\prime} \stackrel{2.4(6) \operatorname{and}(9)}{=}\left((0 \rightarrow(b \rightarrow a)) \rightarrow(b \rightarrow a)^{\prime}\right)^{\prime} \stackrel{2.4(4)}{=}\left(\left((b \rightarrow a)^{\prime} \rightarrow(b \rightarrow\right.\right.$ $\left.a)) \rightarrow(b \rightarrow a)^{\prime}\right)^{\prime} \stackrel{2.2(4)}{=}\left((b \rightarrow a) \rightarrow(b \rightarrow a)^{\prime}\right)^{\prime} \stackrel{2.2(4)}{=}(b \rightarrow a)^{\prime \prime} \stackrel{x^{\prime \prime} \approx x}{=} b \rightarrow a$ $\stackrel{2.4(12)}{=}(a \rightarrow b) \rightarrow(b \rightarrow a)$, completing the proof.

THEOREM 5.15. $42 \mathcal{C} 12=42 \mathcal{C} 13=42 \mathcal{C} 15=42 \mathcal{D} 12=42 \mathcal{F} 35$.

Proof. If $\mathbf{A} \in 42 \mathcal{C} 12$ then, by Lemma 5.13, $\mathbf{A} \models 0 \rightarrow x \approx x \rightarrow x$. Hence, using Theorem 5.14, $\mathbf{A} \models(y)$ for all $(y) \in K$. Consequently $\mathbf{A} \models(42 C 13)$, proving $42 \mathcal{C} 12 \subseteq 42 \mathcal{C} 13$. Similar arguments can be used for the remaining inclusions.

\section{Weak associative laws with length 4 and with 3 variables.}

Possible words of length 4 with 3 variables are:
$\mathrm{A}:\langle x, x, y, z\rangle$,
$\mathrm{D}:\langle x, y, z, x\rangle$,
$\mathrm{B}:\langle x, y, x, z\rangle$,
$\mathrm{E}:\langle x, y, z, y\rangle$,
$\mathrm{C}:\langle x, y, y, z\rangle$,
$\mathrm{F}:\langle x, y, z, z\rangle$.

Ways in which a word of length 4 can be bracketed:
$1: a \rightarrow(a \rightarrow(a \rightarrow a))$,
4: $(a \rightarrow(a \rightarrow a)) \rightarrow a$,
2: $a \rightarrow((a \rightarrow a) \rightarrow a)$,
5: $((a \rightarrow a) \rightarrow a) \rightarrow a$.
3: $(a \rightarrow a) \rightarrow(a \rightarrow a)$, 
Note that these identities are precisely the identities of Bol-Moufang type which were, as mentioned in the Introduction, analysed in [CS18a] with a slightly different notation wherein the first two digits were not used; for example, $43 \mathcal{A} 23$ was denoted by $\mathcal{A}_{23}$, etc.

Recall that the varieties $43 \mathcal{A} 12,43 \mathcal{A} 23$, and $43 \mathcal{A} 23$ are defined, respectively, by the following:

(43A12) $x \rightarrow(x \rightarrow(y \rightarrow z)) \approx x \rightarrow((x \rightarrow y) \rightarrow z)$

(43A23) $x \rightarrow((x \rightarrow y) \rightarrow z) \approx(x \rightarrow x) \rightarrow(y \rightarrow z)$

$(43 \mathrm{~F} 25) x \rightarrow((y \rightarrow z) \rightarrow z) \approx((x \rightarrow y) \rightarrow z) \rightarrow z$.

THEOREM 6.1. [CS18a] There are 4 nontrivial varieties of Bol-Moufang type that are distinct from each other: $\mathcal{S L}, 43 \mathcal{A} 12,43 \mathcal{A} 23$ and $43 \mathcal{F} 25$; and they satisfy the following inclusions:

1. $\mathcal{S} \mathcal{L} \subset 43 \mathcal{A} 23 \subset 43 \mathcal{F} 25$,

2. $\mathcal{S L} \subset 43 \mathcal{A} 12$,

3. $\mathcal{B A} \subset 43 \mathcal{A} 12 \subset 43 \mathcal{F} 25$,

4. $43 \mathcal{F} 25 \subset \mathcal{S}$,

5. $\mathcal{S} \mathcal{L}=43 \mathcal{A} 23 \cap 43 \mathcal{A} 12$.

LEMMA 6.2. $43 \mathcal{F} 25=42 \mathcal{B} 35$.

Proof. Let A $\in$ 42B335. By Lemma 5.9,

$$
\mathbf{A} \models 0 \rightarrow(x \rightarrow x) \approx x \rightarrow x .
$$

Hence, using Lemmas 2.8 and 2.9, we have

$$
\mathbf{A}=(x \rightarrow x) \rightarrow y^{\prime} \approx((x \rightarrow x) \rightarrow y)^{\prime} .
$$

Let $a, b, c \in A$. Then $((a \rightarrow b) \rightarrow c) \rightarrow c \stackrel{(I)}{=}\left(\left(c^{\prime} \rightarrow a\right) \rightarrow(b \rightarrow c)^{\prime}\right)^{\prime} \rightarrow c$ $\stackrel{2.7(2)}{=} c^{\prime} \rightarrow\left(\left(c^{\prime} \rightarrow a\right) \rightarrow(b \rightarrow c)^{\prime}\right)^{\prime \prime} \stackrel{x^{\prime \prime} \approx x}{=} c^{\prime} \rightarrow\left(\left(c^{\prime} \rightarrow a\right) \rightarrow(b \rightarrow c)^{\prime}\right)$ $\stackrel{2.7(2)}{=} c^{\prime} \rightarrow\left((b \rightarrow c) \rightarrow\left(c^{\prime} \rightarrow a\right)^{\prime}\right) \stackrel{2.7(1)}{=}(b \rightarrow c) \rightarrow\left(c^{\prime} \rightarrow\left(c^{\prime} \rightarrow a\right)^{\prime}\right) \stackrel{2.7(2)}{=}(b \rightarrow$ $c) \rightarrow\left(\left(c^{\prime} \rightarrow a\right) \rightarrow c\right) \stackrel{(I)}{=}(b \rightarrow c) \rightarrow\left(\left(c^{\prime} \rightarrow c^{\prime}\right) \rightarrow(a \rightarrow c)^{\prime}\right)^{\prime} \stackrel{2.7(2)}{=}(b \rightarrow c) \rightarrow$ $\left((c \rightarrow c) \rightarrow(a \rightarrow c)^{\prime}\right)^{\prime} \stackrel{(6.2)}{=}(b \rightarrow c) \rightarrow((c \rightarrow c) \rightarrow(a \rightarrow c))^{\prime \prime} \stackrel{x^{\prime \prime} \approx x}{=}(b \rightarrow c) \rightarrow$ 
$((c \rightarrow c) \rightarrow(a \rightarrow c)) \stackrel{2.4(16)}{=}(b \rightarrow c) \rightarrow(a \rightarrow c) \stackrel{2.7(1)}{=} a \rightarrow((b \rightarrow c) \rightarrow c)$.

Hence, $\mathbf{A} \models(43 F 25)$ and consequently,

$42 \mathcal{B} 35 \subseteq 43 \mathcal{F} 25$

Assume now that $\mathbf{A} \in 43 \mathcal{F} 25$ and $a \in A$. Then $0 \rightarrow(a \rightarrow a) \stackrel{2.2(1)}{=} 0 \rightarrow$ $\left(\left(0^{\prime} \rightarrow a\right) \rightarrow a\right) \stackrel{(43 F 25)}{=}\left(\left(0 \rightarrow 0^{\prime}\right) \rightarrow a\right) \rightarrow a \stackrel{2.2(4)}{=}\left(0^{\prime} \rightarrow a\right) \rightarrow a \stackrel{2.2(1)}{=} a \rightarrow a$. Hence, using Lemma 2.9, we get

$$
\mathbf{A} \models(x \rightarrow x) \rightarrow(y \rightarrow z) \approx((x \rightarrow x) \rightarrow y) \rightarrow z
$$

Using (6.3) we have that $\mathbf{A} \models(42 B 35)$. This completes the proof.

LEMMA 6.3. $43 \mathcal{A} 12=42 \mathcal{C} 12$.

Proof. Let $\mathbf{A} \in 43 \mathcal{A} 12$ and $a \in A$. Observe that $a \rightarrow a \stackrel{2.7(2)}{=} a^{\prime} \rightarrow a^{\prime}$ $\stackrel{x \approx x^{\prime \prime}}{=} a^{\prime} \rightarrow a^{\prime \prime \prime}=a^{\prime} \rightarrow\left(\left(a^{\prime} \rightarrow 0\right) \rightarrow 0\right) \stackrel{(43 A 12)}{=} a^{\prime} \rightarrow\left(a^{\prime} \rightarrow(0 \rightarrow 0)\right) \stackrel{2.4(7)}{=}$ $a^{\prime} \rightarrow(0 \rightarrow 0)=a^{\prime} \rightarrow 0^{\prime} \stackrel{2.7(2)}{=} 0 \rightarrow a$. Hence, by Theorem 5.14, $\mathbf{A} \in 42 \mathcal{C} 12$, implying $43 \mathcal{A} 12 \subseteq 42 \mathcal{C} 12$.

Conversely, take $\mathbf{A} \in 42 \mathcal{C} 12$ and $a, b, c \in A$. By Lemma 5.13,

$$
\mathbf{A} \models 0 \rightarrow x \approx x \rightarrow x
$$

Hence, by Lemmas 2.8 and 2.9,

$$
\mathbf{A} \models(x \rightarrow x) \rightarrow y^{\prime} \approx((x \rightarrow x) \rightarrow y)^{\prime}
$$

and

$$
\mathbf{A} \models(x \rightarrow x) \rightarrow(y \rightarrow z) \approx((x \rightarrow x) \rightarrow y) \rightarrow z
$$

Therefore,

$$
\begin{aligned}
& a \rightarrow((a \rightarrow b) \rightarrow c) \stackrel{(I)}{=} a \rightarrow\left(\left(c^{\prime} \rightarrow a\right) \rightarrow(b \rightarrow c)^{\prime}\right)^{\prime} \stackrel{2.7(2)}{=}\left(\left(c^{\prime} \rightarrow a\right) \rightarrow\right. \\
& \left.(b \rightarrow c)^{\prime}\right) \rightarrow a^{\prime} \stackrel{(I)}{=}\left\{\left(a^{\prime \prime} \rightarrow\left(c^{\prime} \rightarrow a\right)\right) \rightarrow\left((b \rightarrow c)^{\prime} \rightarrow a^{\prime}\right)^{\prime}\right\}^{\prime} \stackrel{x \approx x^{\prime \prime}}{=}\{(a \rightarrow \\
& \left.\left.\left(c^{\prime} \rightarrow a\right)\right) \rightarrow\left((b \rightarrow c)^{\prime} \rightarrow a^{\prime}\right)^{\prime}\right\}^{\prime} \stackrel{2.7(1)}{=}\left\{\left(c^{\prime} \rightarrow(a \rightarrow a)\right) \rightarrow\left((b \rightarrow c)^{\prime} \rightarrow a^{\prime}\right)^{\prime}\right\}^{\prime} \\
& \stackrel{2.4(7)}{=}\left\{\left(c^{\prime} \rightarrow\left(c^{\prime} \rightarrow(a \rightarrow a)\right)\right) \rightarrow\left((b \rightarrow c)^{\prime} \rightarrow a^{\prime}\right)^{\prime}\right\}^{\prime} \stackrel{(42 C 12)}{=}\left\{\left(c ^ { \prime } \rightarrow \left(\left(c^{\prime} \rightarrow a\right) \rightarrow\right.\right.\right. \\
& \left.a)) \rightarrow\left((b \rightarrow c)^{\prime} \rightarrow a^{\prime}\right)^{\prime}\right\}^{\prime 2.7(1)}\left\{\left(\left(c^{\prime} \rightarrow a\right) \rightarrow\left(c^{\prime} \rightarrow a\right)\right) \rightarrow\left((b \rightarrow c)^{\prime} \rightarrow a^{\prime}\right)^{\prime}\right\}^{\prime} \\
& \stackrel{(6.5)}{=}\left\{\left(\left(c^{\prime} \rightarrow a\right) \rightarrow\left(c^{\prime} \rightarrow a\right)\right) \rightarrow\left((b \rightarrow c)^{\prime} \rightarrow a^{\prime}\right)\right\}^{\prime \prime} \stackrel{x \approx x^{\prime \prime}}{=}\left(( c ^ { \prime } \rightarrow a ) \rightarrow \left(c^{\prime} \rightarrow\right.\right.
\end{aligned}
$$




$$
\begin{aligned}
& a)) \rightarrow\left((b \rightarrow c)^{\prime} \rightarrow a^{\prime}\right) \stackrel{(6.6)}{=}\left(\left(\left(c^{\prime} \rightarrow a\right) \rightarrow\left(c^{\prime} \rightarrow a\right)\right) \rightarrow(b \rightarrow c)^{\prime}\right) \rightarrow a^{\prime} \\
& \stackrel{(6.5)}{=}\left(\left(\left(c^{\prime} \rightarrow a\right) \rightarrow\left(c^{\prime} \rightarrow a\right)\right) \rightarrow(b \rightarrow c)\right)^{\prime} \rightarrow a^{\prime} \stackrel{2.7(1)}{=}\left(\left(c ^ { \prime } \rightarrow \left(\left(c^{\prime} \rightarrow a\right) \rightarrow\right.\right.\right. \\
& a)) \rightarrow(b \rightarrow c))^{\prime} \rightarrow a^{\prime} \stackrel{(42 C 12)}{=}\left(\left(c^{\prime} \rightarrow\left(c^{\prime} \rightarrow(a \rightarrow a)\right)\right) \rightarrow(b \rightarrow c)\right)^{\prime} \rightarrow a^{\prime} \\
& \stackrel{2.4(7)}{=}\left(\left(c^{\prime} \rightarrow(a \rightarrow a)\right) \rightarrow(b \rightarrow c)\right)^{\prime} \rightarrow a^{\prime} \stackrel{(6.4)}{=}\left(\left(c^{\prime} \rightarrow(0 \rightarrow a)\right) \rightarrow(b \rightarrow\right. \\
& c))^{\prime} \rightarrow a^{\prime} \stackrel{2.7(1)}{=}\left(\left(0 \rightarrow\left(c^{\prime} \rightarrow a\right)\right) \rightarrow(b \rightarrow c)\right)^{\prime} \rightarrow a^{\prime} \stackrel{2.4(6) \text { and }(9)}{=}\left(\left(\left(0 \rightarrow c^{\prime}\right) \rightarrow\right.\right. \\
& (0 \rightarrow a)) \rightarrow(b \rightarrow c))^{\prime} \rightarrow a^{\prime} \stackrel{(6.4)}{=}\left(\left(\left(c^{\prime} \rightarrow c^{\prime}\right) \rightarrow(0 \rightarrow a)\right) \rightarrow(b \rightarrow c)\right)^{\prime} \rightarrow a^{\prime} \\
& \stackrel{2.7(2)}{=}(((c \rightarrow c) \rightarrow(0 \rightarrow a)) \rightarrow(b \rightarrow c))^{\prime} \rightarrow a^{\prime} \stackrel{(6.4)}{=}(((0 \rightarrow c) \rightarrow(0 \rightarrow a)) \rightarrow \\
& (b \rightarrow c))^{\prime} \rightarrow a^{\prime 2.4(6) \stackrel{\operatorname{and}(9)}{=}}((0 \rightarrow(c \rightarrow a)) \rightarrow(b \rightarrow c))^{\prime} \rightarrow a^{\prime} . \\
& \text { So, } a \rightarrow((a \rightarrow b) \rightarrow c) \stackrel{2.7(1)}{=}((c \rightarrow(0 \rightarrow a)) \rightarrow(b \rightarrow c))^{\prime} \rightarrow a^{\prime} \stackrel{2.7(2)}{=} \\
& \left(\left((0 \rightarrow a)^{\prime} \rightarrow c^{\prime}\right) \rightarrow(b \rightarrow c)\right)^{\prime} \rightarrow a^{\prime} \stackrel{2.7(2)}{=}\left(\left((0 \rightarrow a)^{\prime} \rightarrow c^{\prime}\right) \rightarrow\left(c^{\prime} \rightarrow b^{\prime}\right)\right)^{\prime} \rightarrow a^{\prime} \\
& \stackrel{2.4(13)}{=}\left(\left(0 \rightarrow(0 \rightarrow a)^{\prime \prime}\right) \rightarrow\left(c^{\prime} \rightarrow b^{\prime}\right)\right)^{\prime} \rightarrow a^{\prime} \stackrel{x \approx x^{\prime \prime}}{=}\left(( 0 \rightarrow ( 0 \rightarrow a ) ) \rightarrow \left(c^{\prime} \rightarrow\right.\right. \\
& \left.\left.b^{\prime}\right)\right)^{\prime} \rightarrow a^{\prime} \stackrel{2.4(7)}{=}\left((0 \rightarrow a) \rightarrow\left(c^{\prime} \rightarrow b^{\prime}\right)\right)^{\prime} \rightarrow a^{\prime} \stackrel{2.7(2)}{=}((0 \rightarrow a) \rightarrow(b \rightarrow c))^{\prime} \rightarrow a^{\prime} \\
& \stackrel{2.4(11)}{=} a \rightarrow\left\{((0 \rightarrow a) \rightarrow(b \rightarrow c))^{\prime} \rightarrow a^{\prime}\right\} \stackrel{(6.4)}{=} a \rightarrow\{((a \rightarrow a) \rightarrow(b \rightarrow \\
& \left.c))^{\prime} \rightarrow a^{\prime}\right\} \stackrel{(6.5)}{=} a \rightarrow\left\{\left((a \rightarrow a) \rightarrow(b \rightarrow c)^{\prime}\right) \rightarrow a^{\prime}\right\} \stackrel{(I)}{=} a \rightarrow\{(a \rightarrow(a \rightarrow \\
& \left.a)) \rightarrow\left((b \rightarrow c)^{\prime} \rightarrow a^{\prime}\right)^{\prime}\right\}^{\prime} \stackrel{2.4(7)}{=} a \rightarrow\left\{(a \rightarrow a) \rightarrow\left((b \rightarrow c)^{\prime} \rightarrow a^{\prime}\right)^{\prime}\right\}^{\prime} \stackrel{2.7(2)}{=} a \rightarrow \\
& \left\{\left((b \rightarrow c)^{\prime} \rightarrow a^{\prime}\right) \rightarrow(a \rightarrow a)^{\prime}\right\}^{\prime} \stackrel{2.7(2)}{=} a \rightarrow\left\{(a \rightarrow(b \rightarrow c)) \rightarrow(a \rightarrow a)^{\prime}\right\}^{\prime} \\
& \stackrel{2.7(2)}{=} a \rightarrow\left\{(a \rightarrow(b \rightarrow c)) \rightarrow\left(a^{\prime} \rightarrow a^{\prime}\right)^{\prime}\right\}^{\prime} \stackrel{(6.4)}{=} a \rightarrow\{(a \rightarrow(b \rightarrow c)) \rightarrow \\
& \left.\left(0 \rightarrow a^{\prime}\right)^{\prime}\right\}^{\prime} \stackrel{(I)}{=} a \rightarrow\left(((b \rightarrow c) \rightarrow 0) \rightarrow a^{\prime}\right) \stackrel{2.7(2)}{=} a \rightarrow(a \rightarrow(b \rightarrow c)) \text {. Hence } \\
& \mathbf{A} \in 43 \mathcal{A} 12 \text {. }
\end{aligned}
$$

\section{Weak associative laws with length 4 and with 4 variables.}

The only word of length 4 with 4 variables is:

$\mathrm{A}:\langle t, x, y, z\rangle$.

Ways in which a word of length 4 can be bracketed:

1: $a \rightarrow(a \rightarrow(a \rightarrow a))$,

$2: a \rightarrow((a \rightarrow a) \rightarrow a)$,

3: $(a \rightarrow a) \rightarrow(a \rightarrow a)$,

4: $(a \rightarrow(a \rightarrow a)) \rightarrow a$, 
$5:((a \rightarrow a) \rightarrow a) \rightarrow a$.

Let $L$ be the set consisting of the following varieties $44 \mathcal{A} 12,44 \mathcal{A} 13$, $44 \mathcal{A} 14,44 \mathcal{A} 15,44 \mathcal{A} 23,44 \mathcal{A} 24,44 \mathcal{A} 34,44 \mathcal{A} 35$ and $44 \mathcal{A} 45$.

THEOREM 7.1. If $\mathcal{X} \in L$ then $\mathcal{X}=33 \mathcal{A} 12$.

Proof. It is easy to see that $33 \mathcal{A} 12 \subseteq \mathcal{X}$.

Let $\mathbf{A} \in \mathcal{X}$ and let $a, b, c \in A$.

To facilitate a uniform presentation (and to make the proof shorter), we introduce the following notation, where $u_{0}, x_{0}, y_{0}, z_{0} \in A$ :

The notation

$$
\mathcal{X} / u_{0}, x_{0}, y_{0}, z_{0}
$$

denotes the following statement:

"In the identity $(\mathrm{X})$ that defines the variety $\mathcal{X}$, relative to $\mathcal{S}$, if we assign $u:=u_{0}, x:=x_{0}, y:=y_{0}, z:=z_{0}$ (and simplify it using Lemma 2.2 (1) and $\left.x \approx x^{\prime \prime}\right)$, then $\mathbf{A} \models x \rightarrow(y \rightarrow z) \approx(x \rightarrow y) \rightarrow z^{\prime \prime}$.

Hence, we consider the varieties associated with the following statements:
1. $44 \mathcal{A} 12 / 0^{\prime}, \mathrm{a}, \mathrm{b}, \mathrm{c}$;
4. $44 \mathrm{~A} 15 / 0^{\prime}, \mathrm{a}, \mathrm{b}, \mathrm{c}$;
7. $34 \mathcal{A} 12 / 0^{\prime}, \mathrm{a}, \mathrm{b}, \mathrm{c}$;
2. $44 \mathrm{~A} 13 / \mathrm{a}, \mathrm{b}, 0^{\prime}, \mathrm{c}$;
5. $44 \mathcal{A} 23 / 0^{\prime}, \mathrm{a}, \mathrm{b}, \mathrm{c}$;
8. $44 \mathrm{~A} 35 / 0^{\prime}, \mathrm{a}, \mathrm{b}, \mathrm{c}$;
3. $44 \mathcal{A} 14 / 0^{\prime}, \mathrm{a}, \mathrm{b}, \mathrm{c}$;
6. $44 \mathrm{~A} 24 / \mathrm{a}, 0^{\prime}, \mathrm{b}, \mathrm{c}$;
9. $44 \mathcal{A} 45 / \mathrm{a}, \mathrm{b}, \mathrm{c}, 0$.

It is routine to verify that each of the above statements is true, from which we conclude that, for $\mathcal{X} \in L, \mathcal{X} \subseteq 33 \mathcal{A} 12$.

LEMMA 7.2. If $\mathbf{A} \in 31 \mathcal{A} 12 \cup 43 \mathcal{A} 23 \cup 44 \mathcal{A} 25$ then $\mathbf{A} \models 0 \rightarrow x \approx x$.

Proof. First, we will show that if $\mathbf{A} \in 31 \mathcal{A} 12 \cup 43 \mathcal{A} 23 \cup 44 \mathcal{A} 25$, then $\mathbf{A} \models$ $0^{\prime} \approx 0$.

Let $\mathbf{A} \in 31 \mathcal{A} 12$. Then, by Lemma 2.2 (4) and (31A12), $0^{\prime}=0 \rightarrow 0^{\prime}=$ $0 \rightarrow(0 \rightarrow 0)=(0 \rightarrow 0) \rightarrow 0=0^{\prime} \rightarrow 0=0$.

Let $\mathbf{A} \in 43 \mathcal{A} 23$. Then $0 \stackrel{2.2(1)}{=} 0^{\prime} \rightarrow 0 \stackrel{2.2(1)}{=} 0^{\prime} \rightarrow\left(0^{\prime} \rightarrow 0\right)=(0 \rightarrow 0) \rightarrow$ $\left(0^{\prime} \rightarrow 0\right) \stackrel{(43 A 23)}{=} 0 \rightarrow\left(\left(0 \rightarrow 0^{\prime}\right) \rightarrow 0\right) \stackrel{2.2(4)}{=} 0 \rightarrow\left(0^{\prime} \rightarrow 0\right) \stackrel{2.2(1)}{=} 0 \rightarrow 0=0^{\prime}$. 
Let $\mathbf{A} \in 44 \mathcal{A} 25$, Then $0^{\prime}=0 \rightarrow 0 \stackrel{2.2(1)}{=} 0 \rightarrow\left(0^{\prime} \rightarrow 0\right) \stackrel{2.2(4)}{=} 0 \rightarrow((0 \rightarrow$ $\left.\left.0^{\prime}\right) \rightarrow 0\right) \stackrel{(44 A 25)}{=}\left((0 \rightarrow 0) \rightarrow 0^{\prime}\right) \rightarrow 0=\left(0^{\prime} \rightarrow 0^{\prime}\right) \rightarrow 0 \stackrel{2.2(1)}{=} 0^{\prime} \rightarrow 0 \stackrel{2.2(1)}{=} 0$.

Therefore,

$$
\mathbf{A} \models 0 \approx 0^{\prime}
$$

Hence, for $a \in A, 0 \rightarrow a=0^{\prime} \rightarrow a=a$ by Lemma 2.2 (1).

LEMMA 7.3. If $\mathbf{A} \models 0 \rightarrow x \approx x$ then $\mathbf{A} \in 31 \mathcal{A} 12 \cap 43 \mathcal{A} 23 \cap 44 \mathcal{A} 25$.

Proof. Let $a, b, c, d \in A$. Since $a \rightarrow(a \rightarrow a) \stackrel{2.4(7)}{=} a \rightarrow a \stackrel{\text { hyp }}{=}(0 \rightarrow a) \rightarrow a \stackrel{2.4(4)}{=}$ $(a \rightarrow a) \rightarrow a$, we have $\mathbf{A} \models(31 A 12)$. Observe that, by Lemma 2.12 we have that $\mathbf{A}=t_{3}(x, y, z, t) \approx t_{5}(x, y, z, t)$ and $\mathbf{A} \models t_{3}(x, y, z, t) \approx t_{4}(x, y, z, t)$. Hence, $(a \rightarrow a) \rightarrow(b \rightarrow c)=t_{3}(a, a, b, c)=t_{5}(a, a, b, c)=(a \rightarrow b) \rightarrow(a \rightarrow$ $c) \stackrel{2.7(1)}{=} a \rightarrow((a \rightarrow b) \rightarrow c)$ and consequently, the algebra A satisfies (43A23). Also, the algebra satisfies the identity (44A25), since

$$
\begin{aligned}
&((d \rightarrow a) \rightarrow b) \rightarrow c=t_{4}(b, a, d, c)=t_{3}(b, a, d, c)=(a \rightarrow b) \rightarrow(d \rightarrow \\
&c) \stackrel{2.7(1)}{=} d \rightarrow((a \rightarrow b) \rightarrow c),
\end{aligned}
$$

LEMMA 7.4. $31 \mathcal{A} 12=43 \mathcal{A} 23=44 \mathcal{A} 25$

Proof. The proof is immediate from Lemma 7.2 and Lemma 7.3.

\section{MAIN THEOREM}

The purpose of this section is to prove our main theorem. The following proposition will be needed to complete the proof of the main theorem.

In the following proposition we combine all the results obtained so far both in this paper and in [CS18a]. In the latter, each of the 60 varieties defined by the weak associative laws of lenth 4 with 3 variables was shown to be equal to one of the three varieties: $43 \mathcal{A} 12,43 \mathcal{A} 23$, and $43 \mathcal{F} 25$. Therefore, we will need only consider these three varieties with the remaining 95 varieties in the following result.

We recall here that the varieties $42 \mathcal{A} 12,43 \mathcal{A} 12,43 \mathcal{A} 23$, and $43 \mathcal{A} 23$ are defined, respectively, by the following:
$(42 \mathrm{~A} 12) x \rightarrow(x \rightarrow(x \rightarrow y)) \approx x \rightarrow((x \rightarrow x) \rightarrow y)$
$(43 \mathrm{~A} 12) x \rightarrow(x \rightarrow(y \rightarrow z)) \approx x \rightarrow((x \rightarrow y) \rightarrow z)$
(43A23) $x \rightarrow((x \rightarrow y) \rightarrow z) \approx(x \rightarrow x) \rightarrow(y \rightarrow z)$
$(43 \mathrm{~F} 25) x \rightarrow((y \rightarrow z) \rightarrow z) \approx((x \rightarrow y) \rightarrow z) \rightarrow z$. 
PROPOSITION 8.1. Each of the possible 155 weak associative subvarieties of $\mathcal{S}$ is equal to one of the follwing varieties:

$\mathcal{S} \mathcal{L}, 43 \mathcal{A} 12,43 \mathcal{A} 23,42 \mathcal{A} 12,43 \mathcal{F} 25$ and $\mathcal{S}$

Proof. We have

1. $\mathcal{S} \mathcal{L} \stackrel{3.1}{=} \mathcal{L} \mathcal{A} \mathcal{L} \mathcal{T} \stackrel{3.1}{=} \mathcal{F} \mathcal{L} \mathcal{E} \mathcal{3 . 1} \mathcal{R} \mathcal{A} \mathcal{L} \mathcal{T} \stackrel{3.2}{=} 33 \mathcal{A} 12 \stackrel{5.4}{=} 42 \mathcal{A} 14 \stackrel{5.4}{=} 42 \mathcal{B} 14 \stackrel{5.4}{=}$ $42 \mathcal{B} 24 \stackrel{5.4}{=} 42 \mathcal{B} 34 \stackrel{5.4}{=} 42 \mathcal{B} 45 \stackrel{5.4}{=} 42 \mathcal{C} 14 \stackrel{5.4}{=} 42 \mathcal{C} 24 \stackrel{5.4}{=} 42 \mathcal{C} 34 \stackrel{5.4}{=} 42 \mathcal{C} 45 \stackrel{5.4}{=}$ $42 \mathcal{D} 14 \stackrel{5.4}{=} 42 \mathcal{D} 34 \stackrel{5.4}{=} 42 \mathcal{E} 12 \stackrel{5.4}{=} 42 \mathcal{E} 14 \stackrel{5.4}{=} 42 \mathcal{E} 23 \stackrel{5.4}{=} 42 \mathcal{E} 24 \stackrel{5.4}{=} 42 \mathcal{E} 34 \stackrel{5.4}{=}$ $42 \mathcal{E} 35 \stackrel{5.4}{=} 42 \mathcal{E} 45 \stackrel{5.4}{=} 42 \mathcal{F} 15 \stackrel{5.4}{=} 42 \mathcal{F} 23 \stackrel{5.4}{=} 42 \mathcal{F} 24 \stackrel{5.4}{=} 42 \mathcal{F} 45 \stackrel{5.4}{=} 42 \mathcal{G} 23 \stackrel{5.4}{=}$ $42 \mathcal{G} 24 \stackrel{5.4}{=} 42 \mathcal{G} 35 \stackrel{5.4}{=} 42 \mathcal{G} 45 \stackrel{5.2(3)}{=} 42 \mathcal{E} 15 \stackrel{5.3}{=} 42 \mathcal{G} 14 \stackrel{5.3}{=} 42 \mathcal{F} 13 \stackrel{5.3}{=} 42 \mathcal{F} 14 \stackrel{5.3}{=}$ $42 \mathcal{F} 34 \stackrel{7.1}{=} 44 \mathcal{A} 12 \stackrel{7.1}{=} 44 \mathcal{A} 13 \stackrel{7.1}{=} 44 \mathcal{A} 14 \stackrel{7.1}{=} 44 \mathcal{A} 15 \stackrel{7.1}{=} 44 \mathcal{A} 23 \stackrel{7.1}{=} 44 \mathcal{A} 24 \stackrel{7.1}{=}$ $44 \mathcal{A} 34 \stackrel{7.1}{=} 44 \mathcal{A} 35 \stackrel{7.1}{=} 44 \mathcal{A} 45$.

2. $43 \mathcal{A} 12 \stackrel{6.3}{=} 42 \mathcal{C} 12 \stackrel{5.15}{=} 42 \mathcal{C} 13 \stackrel{5.15}{=} 42 \mathcal{C} 15 \stackrel{5.15}{=} 42 \mathcal{D} 12 \stackrel{5.15}{=} 42 \mathcal{F} 35 \stackrel{5.2(3)}{=}$ $42 \mathcal{D} 15 \stackrel{5.3}{=} 42 \mathcal{E} 13 \stackrel{5.3}{=} 42 \mathcal{F} 12 \stackrel{5.3}{=} 42 \mathcal{G} 13$.

3. $43 \mathcal{A} 23 \stackrel{7.4}{=} 31 \mathcal{A} 12 \stackrel{4.2}{=} 41 \mathcal{A} 14 \stackrel{4.1(6)}{=} 41 \mathcal{A} 34 \stackrel{4.1(6)}{=} 41 \mathcal{A} 45 \stackrel{4.1(2)}{=} 41 \mathcal{A} 24 \stackrel{7.4}{=}$ $44 \mathcal{A} 25 \stackrel{5.8}{=} 42 \mathcal{A} 24 \stackrel{5.2(2)}{=} 42 \mathcal{A} 34 \stackrel{5.7}{=} 42 \mathcal{A} 45 \stackrel{5.7}{=} 42 \mathcal{B} 13 \stackrel{5.7}{=} 42 \mathcal{B} 15 \stackrel{5.7}{=} 42 \mathcal{B} 23 \stackrel{5.7}{=}$ $42 \mathcal{B} 25 \stackrel{5.7}{=} 42 \mathcal{D} 24 \stackrel{5.7}{=} 42 \mathcal{D} 45 \stackrel{5.7}{=} 42 \mathcal{F} 25 \stackrel{5.7}{=} 42 \mathcal{G} 15 \stackrel{5.3}{=} 42 \mathcal{G} 34 \stackrel{5.3}{=} 42 \mathcal{G} 12$.

4. $42 \mathcal{A} 12 \stackrel{5.2(2)}{=} 42 \mathcal{A} 13 \stackrel{5.2(2)}{=} 42 \mathcal{A} 15 \stackrel{5.12}{=} 42 \mathcal{D} 23 \stackrel{5.12}{=} 42 \mathcal{D} 35$.

5. $43 \mathcal{F} 25 \stackrel{6.2}{=} 42 \mathcal{B} 35 \stackrel{5.11}{=} 42 \mathcal{B} 12 \stackrel{5.11}{=} 42 \mathcal{C} 23 \stackrel{5.11}{=} 42 \mathcal{C} 25 \stackrel{5.11}{=} 42 \mathcal{C} 35 \stackrel{5.11}{=} 42 \mathcal{G} 25 \stackrel{5.3}{=}$ $42 \mathcal{D} 13$.

6. $\mathcal{S} \stackrel{4.1(4)}{=} 41 \mathcal{A} 23 \stackrel{4.1(5)}{=} 41 \mathcal{A} 13 \stackrel{4.1(5)}{=} 41 \mathcal{A} 15 \stackrel{4.1(5)}{=} 41 \mathcal{A} 35 \stackrel{4.1(3)}{=} 41 \mathcal{A} 25 \stackrel{4.1(1)}{=}$ $41 \mathcal{A} 12 \stackrel{5.2(1)}{=} 42 \mathcal{A} 23 \stackrel{5.2(1)}{=} 42 \mathcal{A} 25 \stackrel{5.2(1)}{=} 42 \mathcal{A} 35 \stackrel{5.2(1)}{=} 42 \mathcal{D} 25 \stackrel{5.2(1)}{=} 42 \mathcal{E} 25$.

We are now ready to present the main theorem of this paper.

\section{THEOREM 8.2.}

(a) The following are the 6 varieties defined, relative to $\mathcal{S}$, by the 155 weak associative laws of length $m \leq 4$ that are distinct from each other:

$\mathcal{S} \mathcal{L}, 43 \mathcal{A} 12,43 \mathcal{A} 23,42 \mathcal{A} 12,43 \mathcal{F} 25$ and $\mathcal{S}$ 
(b) They satisfy the following relationships:

1. $\mathcal{S} \mathcal{L} \subset 43 \mathcal{A} 23 \subset 43 \mathcal{F} 25 \subset \mathcal{S}$,

2. $\mathcal{S L} \subset 43 \mathcal{A} 12 \subset 42 \mathcal{A} 12 \subset \mathcal{S}$,

3. $\mathcal{B A} \subset 43 \mathcal{A} 12 \subset 43 \mathcal{F} 25$,

4. $43 \mathcal{A} 12 \nsubseteq 43 \mathcal{A} 23$ and $43 \mathcal{A} 23 \nsubseteq 43 \mathcal{A} 12$,

5. $42 \mathcal{A} 12 \nsubseteq 43 \mathcal{F} 25$ and $43 \mathcal{F} 25 \nsubseteq 42 \mathcal{A} 12$,

6. $43 \mathcal{A} 23 \nsubseteq 42 \mathcal{A} 12$ and $42 \mathcal{A} 12 \nsubseteq 43 \mathcal{A} 23$.

Proof. We first prove (b):

1. Follows from Theorem 6.1.

2. The statement $\mathcal{S} \mathcal{L} \subset 43 \mathcal{A} 12$ follows from Theorem 6.1. Then it remains to check that $43 \mathcal{A} 12 \subset 42 \mathcal{A} 12 \subset \mathcal{S}$.

Let $\mathbf{A} \in 43 \mathcal{A} 12$ and $a, b \in A$. By Proposition 8.1 (2), $\mathbf{A} \in 42 \mathcal{C} 12$. Observe that

$$
42 \mathcal{C} 12 \models 0 \rightarrow x \approx x \rightarrow x
$$

by Lemma 5.13. Then, using Lemma 2.8 (1), we have $42 \mathcal{C} 12 \models 0 \rightarrow$ $(x \rightarrow x) \approx x \rightarrow x$. Hence, by Lemma 2.9 (1),

$$
\mathbf{A} \models(x \rightarrow x) \rightarrow y^{\prime} \approx((x \rightarrow x) \rightarrow y)^{\prime} .
$$

Hence $a \rightarrow((a \rightarrow a) \rightarrow b) \stackrel{2.7(1)}{=}(a \rightarrow a) \rightarrow(a \rightarrow b) \stackrel{2.7(2)}{=}(a \rightarrow a) \rightarrow$ $\left(b^{\prime} \rightarrow a^{\prime}\right) \stackrel{x \approx x^{\prime \prime}}{=}\left((a \rightarrow a) \rightarrow\left(b^{\prime} \rightarrow a^{\prime}\right)\right)^{\prime \prime} \stackrel{(8.2)}{=}\left((a \rightarrow a) \rightarrow\left(b^{\prime} \rightarrow a^{\prime}\right)^{\prime}\right)^{\prime}$ $\stackrel{2.7(2)}{=}\left(\left(a^{\prime} \rightarrow a^{\prime}\right) \rightarrow\left(b^{\prime} \rightarrow a^{\prime}\right)^{\prime}\right)^{\prime} \stackrel{(8.1)}{=}\left(\left(0 \rightarrow a^{\prime}\right) \rightarrow\left(b^{\prime} \rightarrow a^{\prime}\right)^{\prime}\right)^{\prime} \stackrel{2.7(2)}{=}$ $\left(\left(a \rightarrow 0^{\prime}\right) \rightarrow\left(b^{\prime} \rightarrow a^{\prime}\right)^{\prime}\right)^{\prime} \stackrel{(I)}{=}\left(0^{\prime} \rightarrow b^{\prime}\right) \rightarrow a^{\prime} \stackrel{2.2(1)}{=} b^{\prime} \rightarrow a^{\prime} \stackrel{2.7(2)}{=} a \rightarrow b$ $\stackrel{2.4(7)}{=} a \rightarrow(a \rightarrow(a \rightarrow b))$. Thus, $\mathbf{A} \in 42 \mathcal{A} 12$. The following examples show, respectively, that $42 \mathcal{A} 12 \neq 43 \mathcal{A} 12$ and $42 \mathcal{A} 12 \neq \mathcal{S}$.

\begin{tabular}{r|lll}
$\rightarrow:$ & 0 & 1 & 2 \\
\hline 0 & 2 & 2 & 2 \\
1 & 1 & 1 & 2 \\
2 & 0 & 1 & 2
\end{tabular}

\begin{tabular}{r|llll}
$\rightarrow:$ & 0 & 1 & 2 & 3 \\
\hline 0 & 0 & 1 & 2 & 3 \\
1 & 2 & 3 & 2 & 3 \\
2 & 1 & 1 & 3 & 3 \\
3 & 3 & 3 & 3 & 3
\end{tabular}


3. Follows from Theorem 6.1.

4. The algebra $\mathbf{2}_{\mathbf{b}}$ shows that $43 \mathcal{A} 12 \nsubseteq 43 \mathcal{A} 23$, whilst the following algebra shows that $43 \mathcal{A} 23 \nsubseteq 43 \mathcal{A} 12$.

\begin{tabular}{r|rrrr}
$\rightarrow:$ & 0 & 1 & 2 & 3 \\
\hline 0 & 0 & 1 & 2 & 3 \\
1 & 2 & 3 & 2 & 3 \\
2 & 1 & 1 & 3 & 3 \\
3 & 3 & 3 & 3 & 3
\end{tabular}

5. The following algebras show that $42 \mathcal{A} 12 \nsubseteq 43 \mathcal{F} 25$ and $43 \mathcal{F} 25 \nsubseteq 42 \mathcal{A} 12$, respectively.

\begin{tabular}{r|rrr}
$\rightarrow:$ & 0 & 1 & 2 \\
\hline 0 & 2 & 2 & 2 \\
1 & 1 & 1 & 2 \\
2 & 0 & 1 & 2
\end{tabular}

\begin{tabular}{r|rrrr}
$\rightarrow:$ & 0 & 1 & 2 & 3 \\
\hline 0 & 0 & 1 & 2 & 3 \\
1 & 2 & 3 & 2 & 3 \\
2 & 1 & 1 & 3 & 3 \\
3 & 3 & 3 & 3 & 3
\end{tabular}

6. The following algebras show that $43 \mathcal{A} 23 \nsubseteq 42 \mathcal{A} 12$ and $42 \mathcal{A} 12 \nsubseteq 43 \mathcal{A} 23$, respectively.

\begin{tabular}{r|rrrr}
$\rightarrow:$ & 0 & 1 & 2 & 3 \\
\hline 0 & 0 & 1 & 2 & 3 \\
1 & 2 & 3 & 2 & 3 \\
2 & 1 & 1 & 3 & 3 \\
3 & 3 & 3 & 3 & 3
\end{tabular}

\begin{tabular}{r|rr}
$\rightarrow:$ & 0 & 1 \\
\hline 0 & 1 & 1 \\
1 & 0 & 1
\end{tabular}

The proof of the theorem is now complete since (a) is an immediate consequence of Proposition 8.1 and (b).

The Hasse diagram of the poset of the weak associative subvarieties of $\mathcal{S}$ of length $\leq 4$, together with the variety $\mathcal{B} \mathcal{A}$ is given below: 


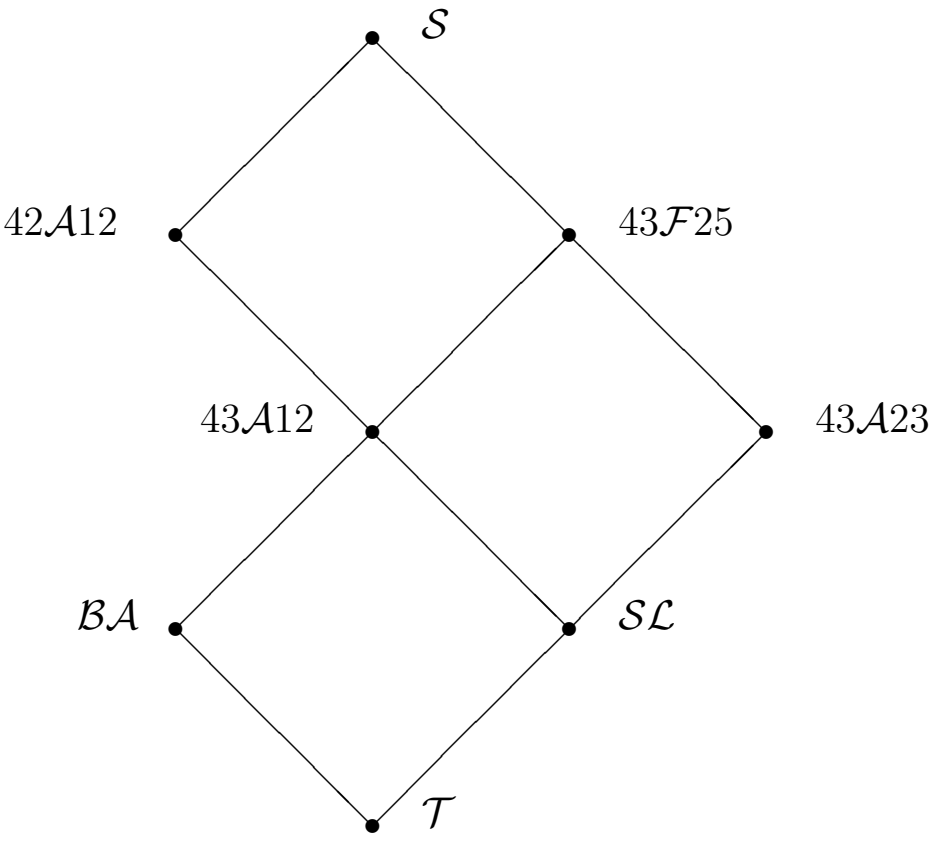

We conclude the paper with the remark that it would be of interest to investigate the weak associative identities, and in particular, the identities of Bol-Moufang type, relative to $\mathcal{I}$ and other (important) subvarieties of $\mathcal{I}$.

Acknowledgment: The first author wants to thank the institutional support of CONICET (Consejo Nacional de Investigaciones Científicas y Técnicas). The authors wish to express their indebtedness to the two anonymous referees for their careful reading of the paper and for their useful suggestions that helped improve the final presentation of this paper.

\section{Compliance with Ethical Standards:}

Conflict of Interest: The first author declares that he has no conflict of interest. The second author declares that he has no conflict of interest. 
Ethical approval: This article does not contain any studies with human participants or animals performed by any of the authors.

Funding: The work of Juan M. Cornejo was supported by CONICET (Consejo Nacional de Investigaciones Cientificas y Tecnicas) and Universidad Nacional del Sur. Hanamantagouda P. Sankappanavar did not receive any specific grant from funding agencies in the public, commercial, or not-for-profit sectors.

\section{References}

[BD74] Balbes R, Dwinger, PH (1974) Distributive lattices. Univ. of Missouri Press, Columbia.

[Be34] Bernstein BA (1934) A set of four postulates for Boolean algebras in terms of the implicative operation. Trans. Amer. Math. Soc. 36 , 876-884.

[BS81] Burris S, Sankappanavar HP (1981) A Course in Universal Algebra. Springer-Verlag, New York. The free, corrected version (2012) is available online as a PDF file at math.uwaterloo.ca/ snburris.

[CS16a] Cornejo JM, Sankappanavar HP (2016) On implicator groupoids. Algebra Univers. $77(2), 125-146$.

doi:10.1007/s00012-017-0429-0. arXiv:1509.03774.

[CS16b] Cornejo JM, Sankappanavar HP (2016) Order in implication zroupoids. Stud Logica, 104(3), 417-453 (2016). doi: 10.1007/s11225-015-9646-8.

[CS16c] Cornejo JM, Sankappanavar HP (2016) Semisimple varieties of implication zroupoids. Soft Comput. 20, 3139-3151. doi: 10.1007/s00500-015-1950-8.

[CS17] Cornejo JM, Sankappanavar HP (2017) On derived algebras and subvarieties of implication zroupoids. Soft Comput. 21(23), 6963 - 6982. doi: 10.1007/s00500-016-2421-6.

[CS18a] Cornejo JM, Sankappanavar HP (2018) Symmetric implication zroupoids and the identities of Bol-Moufang type. Soft Comput. 22(13), 4319 - 4333. doi: 10.1007/s00500-017-2869-z.

[CS18b] Cornejo JM, Sankappanavar HP (2018) Varieties of implication zroupoids I. (In preparation). 
[CS18c] Cornejo JM, Sankappanavar HP (2018) Implication zroupoids and the identities of associative type, Quasigroups and Related Systems 26, 1334.

[Fe69] Fenyves F (1969) Extra loops. II. Publ. Math. Debrecen 16, 187-192.

[GSV] Gusev SV, Sankappanavar HP and Vernikov BM (2018). The lattice of varieties of implication semigroups. Submitted.

[Ku96] Kunen K (1996) Quasigroups, loops, and associative laws. J. Algebra 185, 194-204. doi: 10.1006/jabr.1996.0321.

[Mc] McCune W (2005-2010) Prover9 and Mace4. http://www.cs.unm.edu/ mccune/prover9/

[PV05a] Phillips JD, Vojtechovsky (2005) The varieties of loops of Bol-Moufang type, Algebra Univers. 54, 259-271. DOI 10.1007/s00012-005-1941-1.

[PV05b] Phillips JD, Vojtechovsky (2005) The varieties of quasigroups of BolMoufang type: an equational reasoning approach. J. Algebra 293, 17-33.

[P167] Płonka J (1967) On distributive quasilattices. Fund. Math. 60, 191-200

[R74] Rasiowa H (1974) An algebraic approach to non-classical logics. NorthHolland, Amsterdam.

[San12] Sankappanavar HP (2012) De Morgan algebras: new perspectives and applications. Sci. Math. Jpn. 75(1): 21-50.

Juan M. Cornejo

Departamento de Matemática

Universidad Nacional del Sur

Alem 1253, Bahía Blanca, Argentina

INMABB - CONICET

Email: jmcornejo@uns.edu.ar

Hanamantagouda P. Sankappanavar

Department of Mathematics

State University of New York

New Paltz, New York 12561

U.S.A.

Email: sankapph@newpaltz.edu 